\title{
SPECTRAL ANALYSIS OF A MODEL OF THE COMPRESSIBLE EULER EQUATIONS*
}

\author{
THADDEUS J. EDENS ${ }^{\dagger}$
}

\begin{abstract}
We construct degenerate quadratic models for the Euler equations which distinguish the stabilizing effect of an antisymmetry in the Lie structure of the Euler equations when this antisymmetry is accounted for versus when it is not. We derive a matrix, depending only on the mesh size, $N$, and the 2-wave strengths, whose powers propagate the 1- and 3-waves up to time $t$. We give sharp estimates for the magnitude of the largest eigenvalue of this matrix and conclude that the solution will decay for initial data of arbitrarily large total variation, of order the 4-th root of $N$ in the limit $N$ approaches infinity, when the antisymmetry is accounted for, and only for sufficiently small total variation when it is not.
\end{abstract}

Key words. hyperbolic conservation laws, spectral analysis

Subject classifications. 35L65, 76N15

\section{Introduction}

We consider the Cauchy problem for the general $n \times n$ hyperbolic system of conservation laws,

$$
\begin{aligned}
u_{t}+f(u)_{x} & =0, \quad u \in \mathbf{R}^{n}, \quad f: \mathbf{R}^{n} \rightarrow \mathbf{R}^{n}, \\
u(x, 0) & =u_{0}(x) .
\end{aligned}
$$

It is well known that, in general, shocks form in solutions to (1.1) in finite time, when approaching characteristics of a single family meet. Once shocks have formed, they will interact, sorting themselves out as the slower waves cross faster waves. Genuine non-linearity in a characteristic family acts to stabilize the solution because approaching waves of the same family combine destructively, and this process reduces the total wave strength. Counteracting this, the geometric nonlinearity resulting from noncommuting charactistic fields produces new waves of other families when two waves interact - and this has a destabilizing effect, the magnitude of which depends on the geometric Lie algebra structure of the non-commuting vector fields.

The purpose of this paper is to investigate the stabilizing effect of a certain antisymmetry in the Lie algebra structure of the Euler equations, a 3-by-3 hyperbolic system of conservation laws. To do this, we construct the simplest model, (based on the degenerate quadratic model [13]), which distinguishes the stabilizing effect when the antisymmetry is accounted for versus when it is not. The results prove that, in the model, solutions with arbitrarily large total variation will eventually decay when the antisymmetry effect is accounted for, but that Glimm's result is sharp, (decay only occurs when the total variation is sufficiently small), for bounded solutions in the case when the antisymmetry effect is neglected. Before describing the model in detail, we first motivate the construction.

In his celebrated paper of 1965 [5], Glimm proved that when each field is either genuinely non-linear or linearly degenerate, and the total variation of the initial data is small, the production of new waves is limited and the solution exists for all time with bounded total variation. Glimm's theorem can be stated as follows, [5]:

${ }^{*}$ Received: September 15, 2006; accepted (in revised version): January 10, 2007. Communicated by Alberto Bressan.

${ }^{\dagger}$ Department of Mathematics, University of Michigan, Ann Arbor, MI 48109, USA (tedens@ sympatico.ca). 
TheOREM 1.1 (Glimm 1965.). Let $\bar{u} \in \mathbf{R}^{n}$ such that (1.1) is strictly hyperbolic in a neighborhood of $\bar{u}$. There exist positive constants $C$ and $V_{\text {crit }}$, depending only on the flux function $f$ in a neighborhood of $\bar{u}$, such that whenever the initial data $u_{0}(x)$ satisfies,

$$
\left|u_{0}-\bar{u}\right|_{\infty}<V_{\text {crit }} \quad \text { and } \quad T V\left\{u_{0}(\cdot)\right\}<V_{\text {crit }},
$$

then a global weak solution with shocks exists for all time and

$$
T V\{u(\cdot, t)\}<C \cdot T V\left\{u_{0}(\cdot)\right\} .
$$

Much work has been done on uniqueness, regularity and decay of the Glimm scheme solutions and limits under vanishing viscosity $[3,2,6,7,9,10]$. We restrict our discussion to solutions having large initial total variation, a situation to which Glimm's theorem does not apply. In particular, for the Euler equations of gas dynamics in one dimension,

$$
\begin{aligned}
\rho_{t}+(\rho v)_{x} & =0, \\
(\rho v)_{t}+\left(\rho v^{2}+p\right)_{x} & =0, \\
E_{t}+((E+p) v)_{x} & =0,
\end{aligned}
$$

a $3 \times 3$ hyperbolic system, it is not known whether solutions remain bounded for all time when initial total variation is large, even when the supnorm is small. In [13], Temple and Young gave the first proof of existence of solutions of (1.2) for large total variation, but the time of existence depended on the supnorm, with an exponential growth rate. The proof was based on getting estimates for a degenerate quadratic model that neglected the effect of the genuine nonliearity, yet preserved the geometric nonlinearity at the leading (quadratic) level. However, the analysis failed to account for a certain antisymmetry in the Lie Bracket structure constants, (an antisymmetry between different wave families, which is in addition to the natural antisymmetry of a Lie algebra). In [17], Young applied eigenvalue analysis to a modified degenerate quadratic model with periodic initial data in order to account for this antisymmetry of the Lie algebra. The results prove that, for periodic data, solutions are globally bounded. The purpose here is to get time independent bounds for the exact degenerate quadratic model introduced in [13], in the presence of large total variation. Before we state the theorem, we first recall the derivation of the degenerate quadratic model in [13].

Recall that system (1.1) is strictly hyperbolic in a neighborhood $U$ of a point $\bar{u} \in \mathbf{R}^{n}$ if the Jacobian matrix, $\mathrm{d} f$, has distinct real eigenvalues throughout $U$. We denote the eigenvalues at the point $u$ by $\lambda_{1}(u)<\ldots<\lambda_{n}(u)$. For hyperbolic systems, the matrix $\mathrm{d} f(u)$ has a complete set of eigenvectors. We denote these by $r_{i}(u)$, $i=1, \ldots, n$, so that

$$
\mathrm{d} f(u) r_{i}(u)=\lambda_{i}(u) r_{i}(u) .
$$

The functions $r_{i}(u)$ are referred to as characteristic fields. We restrict discussion to systems for which each characteristic field is either genuinely nonlinear,

$$
\nabla \lambda_{i} \cdot r_{i} \neq 0,
$$


or linearly degenerate,

$$
\nabla \lambda_{i} \cdot r_{i}=0
$$

throughout $U$. Each characteristic field is normalized to unit length and the direction is chosen such that,

$$
\nabla \lambda_{i} \cdot r_{i} \geq 0
$$

The Riemann problem for (1.1) has initial data of the form

$$
u(x, 0)=\left\{\begin{array}{lll}
u_{L} & : & x<0 \\
u_{R} & : & x>0 .
\end{array}\right.
$$

A unique solution, constructed by Lax, exists when $u_{L}$ and $u_{R}$ lie in $U$ and consists of at most $n+1$ constant states separated by rarefaction fans, shock waves and contact discontinuities. We denote the solution by $\left\langle u_{0}, u_{1}, \ldots, u_{n}\right\rangle$ where the $u_{i}$ are the constant states and $u_{0}=u_{L}$ and $u_{n}=u_{R}$. For each $i$ the pair of adjacent states $u_{i-1}$ and $u_{i}$ is separated by an $i$-wave, and the state $u_{i}$ lies on either the integral curve of $r_{i}$ starting at $u_{i-1}$ or on the $i$-shock curve starting at $u_{i-1}$. These two curves have second order tangency at $u_{i-1}$. We denote the solution in terms of wave strengths, as constructed in [14], by $\left\langle\epsilon_{1}, \ldots, \epsilon_{n}>\right.$.

The Glimm scheme and front tracking methods, [5, 1, 4, 11], are schemes for implementing wave interactions. For our purposes we discuss the Glimm scheme. The Glimm scheme pieces together solutions to Riemann problems to build an approximate solution to (1.1) on $\mathbf{R} \times \mathbf{R}_{\geq 0}$. Fix $\Delta x>0$ and $\Delta t>0$ and let $x_{i}=i \Delta x, i \in \mathbf{Z}$ and $t_{j}=j \Delta t, j \in \mathbf{Z}_{\geq 0}$ be partitions of $\mathbf{R}$ and $\mathbf{R}_{\geq 0}$, respectively. Given an initial condition $u(x, 0) \equiv u(x)$, we approximate $u(x)$ by a piecewise constant function which is constant on intervals $\left(x_{i}, x_{i}+1\right)$. Suppose now that we have an approximate solution at some time $t_{j}$. We then solve the Riemann problem for (1.1) on each rectangle, $\left[x_{i-1 / 2}, x_{i+1 / 2}\right] \times\left[t_{j}, t_{j+1}\right]$. We specify the solution at time $t_{j+1}$ as follows. We randomly choose a number $\theta$ in $[-1,1]$ and set, $u\left(x, t_{j+1}\right)=u\left(x_{i}+\theta \Delta x / 2, t_{j+1}^{-}\right)$for each $x$, $x_{i-1 / 2}<x<x_{i+1 / 2}$. The left and right states of a Riemann problem at time $t_{j+1}$ come from solutions to adjacent Riemann problems at time $t_{j}$. We can view this as follows. Some of the waves from each of the left and right Riemann solutions are interacting to produce new waves. The waves entering this interaction from the left increase in speed from left-to-right as do the waves entering from the right, but each wave entering from the left is faster than each wave entering from the right. The solution to the subsequent Riemann problem has waves increasing in speed from left-to-right. Thus the interacting waves sort themselves out by speed.

The strengths of the waves after an interaction can be expressed in terms of the strengths of the interacting waves. Denote the strengths of the waves entering from the left and right by $\left\langle\eta_{1}, \ldots, \eta_{n}\right\rangle$ and $\left\langle\theta_{1}, \ldots, \theta_{n}\right\rangle$, respectively, and denote the strengths of the waves after interaction by $\left\langle\epsilon_{1}, \ldots, \epsilon_{n}\right\rangle$. The strenths of the waves after interaction are given by (Young [14])

$$
\epsilon_{i}=\eta_{i}+\theta_{i}+\sum_{j>k} \eta_{j} \theta_{k}\left[r_{j}, r_{k}\right]^{i}+O\left(|\eta|^{3}+|\theta|^{3}\right),
$$

where $\left[r_{j}, r_{k}\right]^{i}$ denotes the $i$-th component of the Lie bracket of the $j$-th and $k$-th characteristic fields. The Lie bracket completely determines the interaction at the 
quadratic level. This quadratic term describes new waves that are created when waves interact. A system with non-vanishing Lie brackets is said to have a geometric nonlinearity.

A Riemann coordinate for a characteristic family is a function, $w: U \rightarrow \mathbf{R}$, such that the left eigenvector of the Jacobian $\mathrm{d} f$ is the gradient of $w$. The $i$-th left eigenvector, $l_{i}$ is defined by

$$
l_{i} \cdot \mathrm{d} f=\lambda_{i} l_{i} .
$$

Thus $w$ is a Riemann coordinate if

$$
l_{i}=\nabla w .
$$

It is well known that for $2 \times 2$ systems the characteristic fields may be rescaled so that the Lie bracket of the two fields vanishes in a neighborhood of a point. This is not the case for larger systems unless each characteristic field has a Riemann coordinate.

Now we can recall Temple and Young's construction of the degenerate quadratic model associated with the Euler equations [13]. Expressed in Lagrangian coordinates, the Euler equations are [12],

$$
\begin{aligned}
v_{t}-w_{x} & =0, \\
w_{t}+p_{x} & =0, \\
E_{t}+(p w)_{x} & =0,
\end{aligned}
$$

where $v=1 / \rho$ is the specific volume. It is well known that the Euler system has three characteristic families, two of which are genuinely non-linear and one of which is linearly degenerate. As a consequence of the Second Law of Thermodynamics, the linearly degenerate field possesses a Riemann coordinate. The characteristic speeds are $\pm c$ and 0 , where $c=\sqrt{p_{\rho}}$ is the sound speed. The first and third characteristic fields, corresponding to speeds $\pm c$, are genuinely nonlinear, while the second characteristic field is linearly degenerate. The following two Lemmas from [13] provide for a simplification of the Lie bracket components of the characteristic fields.

LEMMA 1.2. Let the Lie algebra structure constants $\Lambda_{i}^{j k}$ be defined by

$$
\left[r_{j}, r_{k}\right]=\sum_{i} \Lambda_{i}^{j k} r_{i}
$$

and assume that there exists a Riemann coordinate for the p-th characteristic field. Then we have

$$
\Lambda_{p}^{j k}=0 \quad \text { for allj, } k
$$

Lemma 1.3. Let $\bar{u}$ be as in Theorem 1.1. There exists a normalization of $r_{i}$ such that

$$
\Lambda_{i}^{j k}(\bar{u})=0 \quad \text { for } \quad i=j \quad \text { or } \quad i=k .
$$

The characteristic fields may be rescaled, as a result of Lemmas 1.2 and 1.3, such that the Lie structure coefficients for (1.4) at the point $\bar{u}$ are

$$
\begin{aligned}
\Lambda_{3}^{21}=-\Lambda_{1}^{32} & =1, \\
\Lambda_{i}^{j k} & =0 \text { otherwise. }
\end{aligned}
$$


The minus sign in (1.5) introduces the cancelation in wave interactions, mentioned above, that is not present when absolute values are taken and all $\Lambda_{i}^{j k}$ are estimated as positive, [13]. The purpose of this paper is to compare this model of wave interaction to one not having this antisymmetry; that is, we compare the two models

$$
\begin{aligned}
\Lambda_{3}^{21}=\sigma \Lambda_{1}^{32} & =1, \\
\Lambda_{i}^{j k} & =0 \text { otherwise }
\end{aligned}
$$

where $\sigma \in\{-1,1\}$. We note that Young studied systems having these Lie structure coefficients and for completeness we recall the flux functions of his two systems, [15]. These are

$$
\begin{aligned}
& f_{1}(u, v, w)=\left(\begin{array}{c}
w e^{2 v} \\
0 \\
u e^{-2 v}
\end{array}\right) \quad \sigma=-1, \quad \text { and } \\
& f_{2}(u, v, w)=\left(\begin{array}{c}
w+2 u v \\
0 \\
u\left(1-4 v^{2}\right)-2 v w
\end{array}\right) \quad \sigma=1 .
\end{aligned}
$$

It is assumed in this model that waves travel at constant speed. That is, the effect of genuine nonlinearity is neglected. Further, terms in the wave interaction, equation (1.3), beyond the quadratic level are neglected, so that the wave strengths after interaction are given exactly by

$$
\epsilon_{i}=\eta_{i}+\theta_{i}+\sum_{j>k} \eta_{j} \theta_{k} \Lambda_{i}^{j k} .
$$

We now analyze the degenerate quadratic model in the case of compactly supported initial data comprised of an oscillating wave field of $N+1$ contact discontinuities of constant strength but alternating sign, together with waves of arbitrary sign and strength in the 1- and 3-wave fields. This is the simplest model that distinguishes the stabilizing effect of the antisymmetry, (the minus sign), in the geometric nonlinearity (1.5). For $N>0$ an integer, let $x_{i}=i / N$ for each $i \in \mathbf{Z}$ and let $t_{j}=j /(c N)$ for each $j \in \mathbf{Z}_{\geq 0}$. Let $\alpha_{i}^{k}, \beta_{i}^{k}$, and $\gamma_{i}^{k}$ denote the 1-, 2-, and 3-wave strengths, respectively, at the grid point $\left(x_{i}, t_{k}\right)$. An initial condition for this model problem consists of three wave strengths, $\alpha_{i}^{0}, \beta_{i}^{0}$, and $\gamma_{i}^{0}$, at each initial grid point $\left(x_{i}, t_{0}\right)$. We restrict to initial data that are supported on the unit interval, so that $\alpha_{i}^{0}=\beta_{i}^{0}=\gamma_{i}^{0}=0$ for $i<0$ or $i>N$. According to (1.6) the Lie coefficients $\Lambda_{k}^{13}$ are zero; hence by (1.7) we have that an interaction involving a 1 -wave and a 3 -wave leaves the 2 -waves unchanged and produces no new waves. Hence all changes in wave strengths are a result of interactions that involve a 2-wave. By our choice of grid spacing, all interactions involving a 2 -wave occur at grid points. The solution evolves with the 2 -waves constant for all time, $\beta_{i} \equiv \beta_{i}^{0}=\beta_{i}^{k}$, for each $k>0$, while the 1 - and 3 -waves at grid point $\left(x_{i}, t_{k+1}\right)$ are given in terms of the waves at time-step $k$ by

$$
\begin{aligned}
\alpha_{i}^{k+1} & =\alpha_{i+1}^{k}+\sigma \gamma_{i-1}^{k} \beta_{i}, \\
\gamma_{i}^{k+1} & =\alpha_{i+1}^{k} \beta_{i}+\gamma_{i-1}^{k} .
\end{aligned}
$$

Once a 1-wave or 3 -wave exits the unit interval it continues to propagate without interaction for all future time. It will contribute to the total variation at all future 
times, but it will not affect the solution within the unit interval. We therefore restrict our tracking of waves to those waves that remain within the unit interval, and only consider the waves that exit the unit interval in the estimate of total variation. Equations (1.8) and (1.8) are expressed in matrix notation as

$$
S\left(\alpha_{1}^{k}, \ldots, \alpha_{N}^{k}, \gamma_{0}^{k}, \ldots, \gamma_{N-1}^{k}\right)^{T}=\left(\alpha_{1}^{k+1}, \ldots, \alpha_{N}^{k+1}, \gamma_{0}^{k+1}, \ldots, \gamma_{N-1}^{k+1}\right)^{T}
$$

where the $(2 N) \times(2 N)$ matrix $S$ is given by

$$
S=\left(\begin{array}{cc}
R^{T} & Y \\
X & R
\end{array}\right)
$$

where $X=\operatorname{diag}\left(\beta_{0}, \ldots, \beta_{N-1}\right), Y=\sigma \operatorname{diag}\left(\beta_{1}, \ldots \beta_{N}\right)$ and $R$ is the $N \times N$ right-shift matrix which satisfies $R \mathbf{x}=\left(0, x_{1}, \ldots, x_{N-1}\right)^{T}$ for each $N \times 1$ vector $\mathbf{x}=\left(x_{1}, \ldots, x_{N}\right)^{T}$.

The total variation in the 2 -waves, denoted by $V_{2}$, is equal to the constant value

$$
V_{2}=\sum_{0 \leq i \leq N}\left|\beta_{i}^{0}\right|
$$

for all time. The total variation in the 1 - and 3 -waves at time $t_{k}$, denoted by $V_{13}$ is equal to the sum of the absolute value of each 1 - and 3 -wave at that time. This includes all waves inside the unit interval at time $t_{k}$ as well as each wave that had exited the unit interval at an earlier time. It is given by

$$
V_{13}^{k}=\sum_{0<i \leq N}\left|\alpha_{i}^{k}\right|+\sum_{0 \leq i<N}\left|\gamma_{i}^{k}\right|+\sum_{0 \leq p<k}\left(\left|\alpha_{1}^{p}\right|+\left|\gamma_{N-1}^{p}\right|\right)
$$

and is bounded below and above as follows

$$
\left\|S^{k}\left(\alpha^{\mathbf{0}}, \gamma^{\mathbf{0}}\right)^{\mathbf{T}}\right\|_{1} \leq V_{13}^{k} \leq\left\|S^{k}\left(\alpha^{\mathbf{0}}, \gamma^{\mathbf{0}}\right)^{\mathbf{T}}\right\|_{1}+2 \sum_{0 \leq p<k}\left\|S^{p}\left(\alpha^{\mathbf{0}}, \gamma^{\mathbf{0}}\right)^{\mathbf{T}}\right\|_{\infty}
$$

where $\alpha^{\mathbf{0}}=\left(\alpha_{\mathbf{1}}^{\mathbf{0}}, \ldots, \alpha_{\mathbf{N}}^{\mathbf{0}}\right)$ and $\gamma^{\mathbf{0}}=\left(\gamma_{\mathbf{0}}^{\mathbf{0}}, \ldots, \gamma_{\mathbf{N}-\mathbf{1}}^{\mathbf{0}}\right)$. To understand the behavior of $V_{13}^{k}$ in the limit $k \rightarrow \infty$, consider the spectral radius of the matrix $S$. For any matrix norm, $\|\cdot\|$, the spectral radius of $S$ is defined as

$$
\operatorname{spr} S=\lim _{p \rightarrow \infty}\left\|S^{p}\right\|^{1 / p} .
$$

The spectral radius of a matrix is independent of the norm employed and is also equal to the largest eigenvalue, in modulus, of the matrix. Suppose the scattering matrix $S$ satisfies $\operatorname{spr} S<1$. It follows from (1.9) that for any initial data $\left(\alpha^{\mathbf{0}}, \gamma^{\mathbf{0}}\right)$, the sequence $\left\{V_{13}^{k}\right\}_{k \geq 0}$ is bounded. Conversely, suppose that $\operatorname{spr} S>1$. It follows from (1.9) that there exist initial data $\left(\alpha^{\mathbf{0}}, \gamma^{\mathbf{0}}\right)$ such that the sequence $\left\{V_{13}^{k}\right\}_{k \geq 0}$ is unbounded.

Because rescaling all 1 - and 3 -waves by a constant factor does not affect the asymptotic behavior of $V_{13}^{2}$, the total variation in 1 - and 3-waves can be arbitrarily large for stable systems. The 2 -waves determine the spectral radius and hence they determine whether the system is stable or unstable. Thus our goal is to determine the spectral radius of $S$ as a function of the 2-waves in the degenerate quadratic model. It appears to be a very difficult problem to obtain precise estimates on the largest eigenvalue of $S$ for general 2-wave fields. In this paper we give precise estimates in four special cases which are sufficient to distinguish the effect of the antisymmetry, (the 
sign of $\sigma$ in equation (1.6)), in the geometric nonlinearity. These cases are: $\beta_{i}=\tau^{i} b$, where $\tau \in\{-1,1\}$ and $b>0$, and $\sigma \in\{-1,1\}$. The total variation in 2 -waves in each of these cases is, $V_{2}=(N+1) b$. We denote the scattering matrix in these cases by $S(b)$, though it depends on $\sigma, \tau$ and $N$ as well. The main result of this paper is the following theorem which gives sharp thresholds for decay in the degenerate quadratic wave interaction model.

Theorem 1.4. Let $N$ be an integer, $N \geq 2$, and let $\sigma, \tau \in\{-1,1\}$. Let $b_{*}=\inf \{b>0 \mid \operatorname{spr} S(b) \geq 1\}$. Then

$$
b_{*}= \begin{cases}2^{1 / 4} \pi^{1 / 2} N^{-3 / 4}+O\left(N^{-5 / 4}\right) & \text { for } \sigma=-1, \\ 2 \sin \frac{\pi}{2(2 N+1)} & \text { for } \sigma=1 .\end{cases}
$$

The theorem implies that the degenerate quadratic model, in the case $\sigma=-1$, is stable for 2-wave configurations having arbitrarily large variation and, in the case $\sigma=1$, is stable only for 2 -wave configurations having total variation smaller than a bounded function of $N$. Indeed, when $\sigma=-1$ the system is stable and decays in the total variation norm so long as $b<b_{*}$. This is a large total variation result because the total variation at threshold is

$$
V_{2}=(N+1) b^{*}=(N+1)\left(2^{1 / 4} \pi^{1 / 2} N^{-3 / 4}+O\left(N^{-5 / 4}\right)\right) \rightarrow \infty \quad \text { as } N \rightarrow \infty .
$$

On the other hand, when $\sigma=1$ the system is stable and decays only when the total variation is small because the total variation at threshold is

$$
V_{2}=(N+1) b^{*}=2(N+1) \sin \frac{\pi}{2(2 N+1)} \rightarrow \frac{\pi}{2} \quad \text { as } N \rightarrow \infty
$$

The purpose of this paper is to prove Theorem 1.4. The overall idea of the proof is as follows. We prove, in Section 3, the following Lemma which completely characterizes the spectrum of $S(b)$ as the solution set of a pair of polynomial equations in one complex variable.

Lemma 1.5. Let $N$ be a positive integer. Define the functions $\Phi, \Psi: \mathbf{C} \rightarrow \mathbf{C}$ by

$$
\Phi(\zeta)=\left\{\begin{array}{lll}
\sigma \frac{1}{(N+1)^{2}} & : & \zeta=1 \\
\sigma \frac{\zeta^{N}(1-\zeta)^{2}}{\left(1-\zeta^{N+1}\right)^{2}} & : & \zeta \neq 1
\end{array}\right\} \quad \text { and } \quad \Psi(\zeta)=\left\{\begin{array}{lll}
\tau\left(\frac{N}{N+1}\right)^{2} & : & \zeta=1 \\
\tau \frac{\zeta\left(1-\zeta^{N}\right)^{2}}{\left(1-\zeta^{N+1}\right)^{2}} & : & \zeta \neq 1
\end{array}\right\}
$$

For each $b, \lambda \in \mathbf{C}, \lambda$ is an eigenvalue of the matrix $S(b)$ if and only if there exists $\zeta \in \mathbf{C}$ such that $b^{2}=\Phi(\zeta)$ and $\lambda^{2}=\Psi(\zeta)$.

Each of the functions $\Phi$ and $\Psi$ defined in Lemma 1.5 is meromorphic in the complex plane and has a pole at $\zeta=\exp (2 k \pi i /(N+1))$ for each $k \in\{1, \ldots, N\}$. Note that, because $b$ represents 2 -wave strength, $b$ is a real quantity and that by Lemma 1.5 this is equivalent to the condition $\arg \Phi(\zeta)=0$. It is the dependence of $\arg \Phi(\zeta)$ on $\sigma$ that distinguishes the case $\sigma=-1$ from the case $\sigma=1$ in Theorem 1.4. Lemma 1.5 states that each eigenvalue of $S(b)$ corresponds to a solution to the equation $b^{2}=\Phi(\zeta)$. It appears, however, to be a difficult problem to determine all solutions to 
this equation. We therefore consider the new system of equations,

$$
\begin{aligned}
\arg \Phi(\zeta) & =0, \\
|\Phi(\zeta)| & =b^{2}, \\
|\Psi(\zeta)| & =l^{2}
\end{aligned}
$$

where $b$ and $l$ are real numbers. Equation (1.10a) is equivalent to the condition that $b$ is real, while the pair of equations (1.10a) and (1.10b) is equivalent to the equation $\Phi(\zeta)=b^{2}$. We therefore have the following

Corollary 1.6. Let $b, l \in \mathbf{R}, l>0$. Then matrix $S(b)$ has an eigenvalue of modulus $l$ if and only if (1.10) has a solution $\zeta \in \mathbf{C}$.

In Section 2 we define a map from the $\zeta$-plane to $\mathbf{R}^{2}$ that transforms system (1.10) into a system of equations of two real variables, effectively separating the real and imaginary parts of $\zeta$. The transformed equations, however, involve transcendental functions, and it appears to be a difficult problem to find closed form solutions to this system. While we have found asymptotic expansions for some of the solutions, it is difficult to find an asymptotic expansions for each of the $2 N$ solutions. Because we are unable to determine all solutions, we take another approach. We derive, in Section 3, consequences of system (1.10) that reveal essential information about the geometric ordering in the complex plane of the solutions to each pair of equations $(1.10 \mathrm{a}, 1.10 \mathrm{~b})$ and $(1.10 \mathrm{a}, 1.10 \mathrm{c})$. We then use the geometric ordering of the eigenvalues of $S(b)$ to reduce the problem of finding the largest eigenvalue to that of finding one particular solution to the pair of equations $(1.10 \mathrm{a}, 1.10 \mathrm{c})$ when $l=1$. In Section 4 we construct an asymptotic expansion of this solution and then evaluate $\Phi$ at this solution in order to determine $b_{*}$ and thus complete the proof of Theorem 1.4 in the case $\sigma=-1$. We prove Theorem 1.4 in the case $\sigma=1$ in Section 5. Proofs of intermediate results are found in the Appendix.

\section{The Parametrization}

In this section we prove Lemma 1.5. We then introduce and discuss a natural map from the $\zeta$-plane to $\mathbf{R}^{2}$ under which the transformed equations (1.10) are non-polynomial. This map isolates the constraints on the amplitude and phase of $\zeta$ imposed by (1.10).

Lemma 1.5 is proved as follows. We express the eigenvalue equation for $S(b), \operatorname{det}(\lambda-S(b))=0$, as a recursion relation that reduces to the matrix equation, $\left(M^{N}\right)_{11}=0$, for a $2 \times 2$ matrix $M$ that depends on $\lambda$ and $b{ }^{1} \quad$ We diagonalize $M$, which transforms this last equation into two scalar equations. The first of these is the characteristic equation for $M, \operatorname{det}(\omega-M)=0$, and the second is an equation involving $\lambda$ and the new variable, $\zeta$, which is equal to the ratio of the two eigenvalues of matrix $M$. We show that $b^{2}$ and $\lambda^{2}$ may each be expressed as functions of $\zeta$. That is, $b^{2}=\Phi(\zeta)$ and $\lambda^{2}=\Psi(\zeta)$ for the functions $\Phi$ and $\Psi$ defined in Lemma 1.5.

\section{Proof of Lemma 1.5.}

Proof. Let $\Sigma$ be the $2 N \times 2 N$ permutation matrix defined by $\Sigma\left(\alpha_{1}, \ldots, \alpha_{N}, \gamma_{0}, \ldots \gamma_{N-1}\right)^{T}=\left(\gamma_{N-1}, \alpha_{N}, \gamma_{N-2}, \alpha_{N-1}, \ldots \gamma_{0}, \alpha_{1}\right)^{T}$. The $2 N \times 2 N$ scattering matrix $S$ defined by equation (1.8) is similar via conjugation by $\Sigma$ to the

\footnotetext{
${ }^{1}$ For any matrix $A,(A)_{i j}$ refers to the $(i, j)$-th entry of $A$.
} 
following matrix:

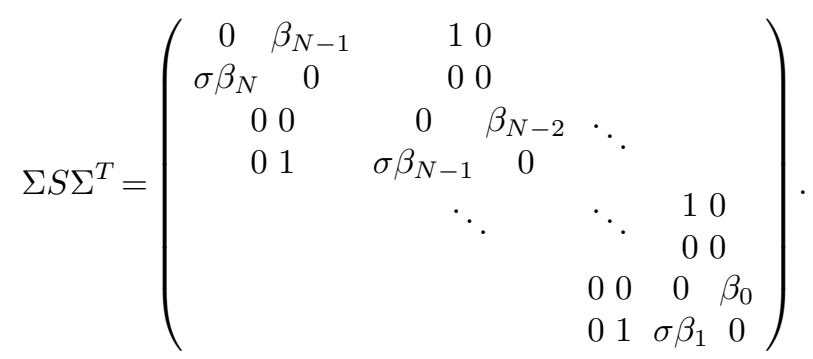

The eigenvalue equation for $S$ is

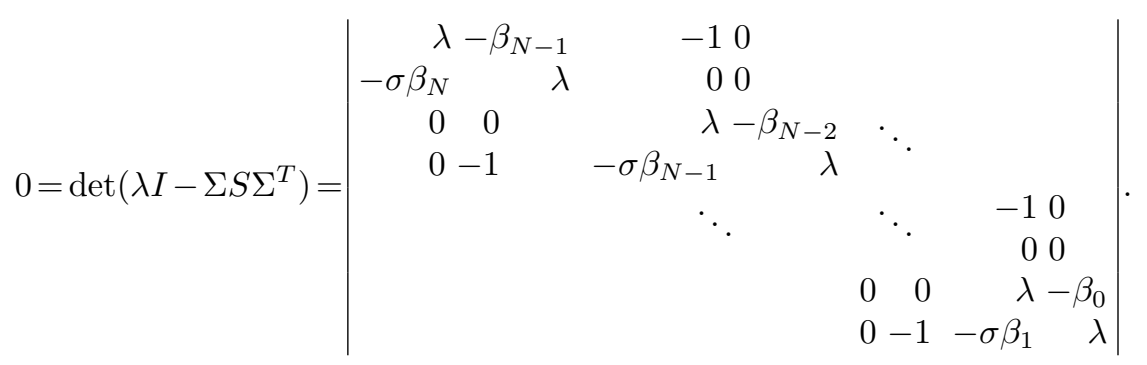

Let $A_{N}=\lambda I-\Sigma S \Sigma^{T}$ and let $B_{N}=\left[A_{N}\right]_{21}$, the matrix formed by deleting the 2 nd row and 1st column from $A_{N}$. Then

$$
\begin{aligned}
& \operatorname{det} A_{N}=\quad \lambda^{2} \operatorname{det} A_{N-1}+\sigma \beta_{N} \operatorname{det} B_{N}, \\
& \operatorname{det} B_{N}=-\beta_{N-1} \operatorname{det} A_{N-1}+\quad \operatorname{det} B_{N-1} .
\end{aligned}
$$

Expressing this in matrix notation we have

$$
\left(\begin{array}{c}
\operatorname{det} A_{N} \\
\operatorname{det} B_{N}
\end{array}\right)=\left(\begin{array}{cc}
\lambda^{2}-\sigma \beta_{N-1} \beta_{N} & \sigma \beta_{N} \\
-\beta_{N-1} & 1
\end{array}\right)\left(\begin{array}{l}
\operatorname{det} A_{N-1} \\
\operatorname{det} B_{N-1}
\end{array}\right) .
$$

Let $\beta_{i}=\tau^{N-i} b$ with $\tau= \pm 1$. We set $\operatorname{det} A_{0}=1$ and $\operatorname{det} B_{0}=0$ and expand (2.1) to find

$$
\begin{aligned}
& 0=\operatorname{det} A_{N}=\left(\begin{array}{ll}
1 & 0
\end{array}\right)\left(\begin{array}{cc}
\lambda^{2}-\sigma \tau b^{2} & \sigma b \\
-\tau b & 1
\end{array}\right)\left(\begin{array}{cc}
\lambda^{2}-\sigma \tau b^{2} & \sigma \tau b \\
-b & 1
\end{array}\right) \cdots \\
& \left(\begin{array}{cc}
\lambda^{2}-\sigma \tau b^{2} & \sigma \tau^{N-1} b \\
-\tau^{N} b & 1
\end{array}\right)\left(\begin{array}{l}
1 \\
0
\end{array}\right) .
\end{aligned}
$$

Next, we multiply (2.2) by $\tau^{N}$ and factor as

$$
\begin{aligned}
0=\left(\begin{array}{ll}
1 & 0
\end{array}\right)\left(\begin{array}{cc}
\tau \lambda^{2}-\sigma b^{2} & \sigma b \\
-b & 1
\end{array}\right)\left(\begin{array}{ll}
1 & 0 \\
0 & \tau
\end{array}\right)^{2}\left(\begin{array}{cc}
\tau \lambda^{2}-\sigma b^{2} & \sigma b \\
-b & 1
\end{array}\right) \cdots \\
\quad\left(\begin{array}{cc}
\tau \lambda^{2}-\sigma b^{2} & \sigma b \\
-b & 1
\end{array}\right)\left(\begin{array}{cc}
1 & 0 \\
0 & \tau^{N}
\end{array}\right)\left(\begin{array}{l}
1 \\
0
\end{array}\right) .
\end{aligned}
$$

We have factored out a diagonal matrix between every other pair of factors in $(2.2)$ and after the last factor when $N$ is odd. Because $\tau^{2}=1$, equation (2.3) simplifies to

$$
0=\left(\begin{array}{ll}
1 & 0
\end{array}\right)\left(\begin{array}{cc}
\tau \lambda^{2}-\sigma b^{2} & \sigma b \\
-b & 1
\end{array}\right)^{N}\left(\begin{array}{l}
1 \\
0
\end{array}\right)
$$


Denote by $M$ the square matrix in (2.4). We want to diagonalize $M$. Let $\rho=\tau \lambda^{2}-\sigma b^{2}$. The eigenvalue equation for $M$ is

$$
\begin{aligned}
0 & =\operatorname{det}(\omega-M)=\left|\begin{array}{cc}
\omega-\rho & -\sigma b \\
b & \omega-1
\end{array}\right| \\
& =\omega^{2}-(1+\rho) \omega+\tau \lambda^{2} .
\end{aligned}
$$

The roots of (2.5) are $\lambda \sqrt{\tau \zeta}$ and $\lambda \sqrt{\tau / \zeta}$, where $\zeta$ satisifies

$$
\lambda \sqrt{\tau \zeta}+\lambda \sqrt{\tau / \zeta}=1+\rho
$$

The matrix $M$ is diagonalized as

$$
\begin{aligned}
& M=\frac{1}{\sigma b(\lambda \sqrt{\tau / \zeta}-\lambda \sqrt{\tau \zeta})}\left(\begin{array}{c}
\sigma b \\
\lambda \sqrt{\tau \zeta}-\rho \lambda \sqrt{\tau / \zeta}-\rho
\end{array}\right) . \\
& \left(\begin{array}{llr}
\lambda \sqrt{\tau \zeta} & \\
& \lambda \sqrt{\tau / \zeta}
\end{array}\right)\left(\begin{array}{cr}
\lambda \sqrt{\tau / \zeta}-\rho & -\sigma b \\
\rho-\lambda \sqrt{\tau \zeta} & \sigma b
\end{array}\right) .
\end{aligned}
$$

Using this factorization, the eigenvalue equation for $S$ is

$$
\begin{aligned}
0=\frac{\tau^{N / 2} \lambda^{N}}{[\sigma b(\lambda \sqrt{\tau / \zeta}-\lambda \sqrt{\tau \zeta})]^{N}}\left(\begin{array}{ll}
1 & 0
\end{array}\right)\left(\begin{array}{cc}
\sigma b & \sigma b \\
\lambda \sqrt{\tau \zeta}-\rho \lambda \sqrt{\tau / \zeta}-\rho
\end{array}\right) & \left(\begin{array}{rr}
\zeta^{N / 2} & \\
& \zeta^{-N / 2}
\end{array}\right)\left(\begin{array}{rr}
\lambda \sqrt{\tau / \zeta}-\rho & -\sigma b \\
\rho-\lambda \sqrt{\tau \zeta} & \sigma b
\end{array}\right)\left(\begin{array}{l}
1 \\
0
\end{array}\right) .
\end{aligned}
$$

This implies that

$$
0=\zeta^{N / 2}(\lambda \sqrt{\tau / \zeta}-\rho)+\zeta^{-N / 2}(\rho-\lambda \sqrt{\tau \zeta})
$$

Eliminating $\rho$ from (2.7) using (2.6) and solving for $\lambda$ we obtain

$$
\lambda=\sqrt{\tau} \frac{\zeta^{N / 2}-\zeta^{-N / 2}}{\zeta^{(N+1) / 2}-\zeta^{-(N+1) / 2}} .
$$

Squaring both sides we obtain

$$
\lambda^{2}=\tau \frac{\zeta\left(1-\zeta^{N}\right)^{2}}{\left(1-\zeta^{N+1}\right)^{2}}
$$

We now solve for $b^{2}$ in terms of $\zeta$. Substituting $\rho=\tau \lambda^{2}-\sigma b^{2}$ into (2.6) and substituting for $\lambda$ from (2.8) and solving for $b^{2}$ we obtain

$$
b^{2}=\sigma \frac{\zeta^{N}(1-\zeta)^{2}}{\left(1-\zeta^{N+1}\right)^{2}} .
$$

Equations (2.9) and (2.10) are exactly the parameterizations we set out to find when $\zeta \in \mathbf{C} \backslash\{1\}$. Functions $f$ and $g$ are continuous at $\zeta=1$. Because the eigenvalues of $S$ depend continuously on $b$, we therefore have that

$$
\lambda^{2}=\tau\left(\frac{N}{N+1}\right)^{2} \quad \text { when } \quad b^{2}=\sigma \frac{1}{(N+1)^{2}} .
$$




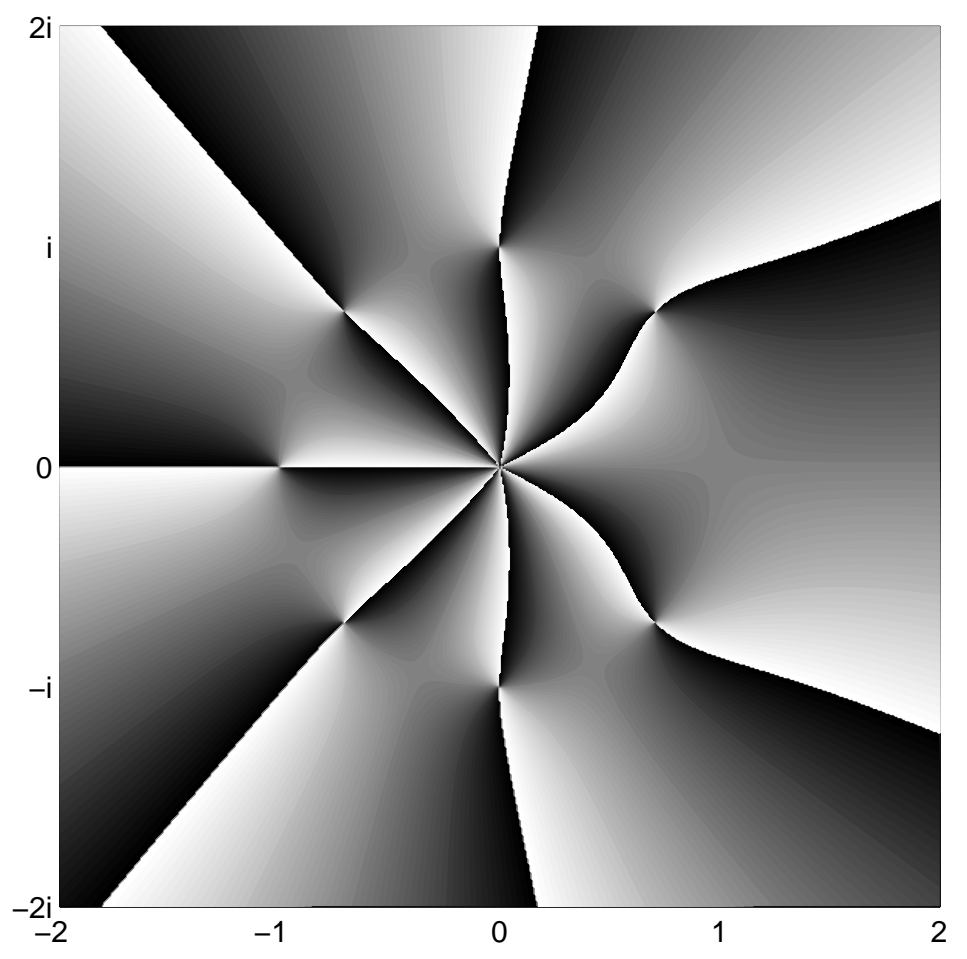

FIG. 2.1. This is a contour plot of $\arg \Phi(\zeta)$ with $N=7$ in the case $\sigma=-1$. The zero contour is visible as the set of curves across which the color jumps from black to white.

Figures 2.1 and 2.2 are contour plots of the function $\arg \Phi(\zeta)$ with $\sigma=-1$ and $\sigma=1$, respectively, and with $N=7$. We have chosen the branch of $\arg \zeta$ which is discontinuous along the positive real axis so that the zero contour is visible as the set of curves across which $\arg \Phi(\zeta)$ jumps from 0 to $2 \pi$ and the color jumps from black to white. This circumvents a problem with contour plots in Mathematica. Had we chosen the standard branch of $\arg \zeta$, which is discontinuous along the negative real axis, then a plot of the zero contour would have also included a plot of the $\pi$ contour. This is a result of the jump in $\arg \zeta$ from $-\pi$ to $\pi$ along the negative real axis. Since 0 lies between these two values, Mathematica plots the negative real axis as part of the zero contour. Figures 2.3 and 2.4 are contour plots of the functions $|\Phi(\zeta)|$ and $|\Psi(\zeta)|$, respectively, with $N=7$. Observe that the level sets of these functions are complicated and consist of one or more closed curves.

We discuss the parametrization given in Lemma 1.5 in detail. The functions $\Phi$ and $\Psi$ possess the following symmetries. Each is invariant under the transformation $\zeta \rightarrow 1 / \zeta$. For each real $b>0$ and each $\zeta$ satisfying $b^{2}=\Phi(\zeta)$ we have

$$
|\Psi(\bar{\zeta})|=|\overline{\Psi(\zeta)}|=|\Psi(\zeta)|,
$$

and

$$
\Phi(\bar{\zeta})=\overline{\Phi(\zeta)}=\Phi(\zeta)
$$




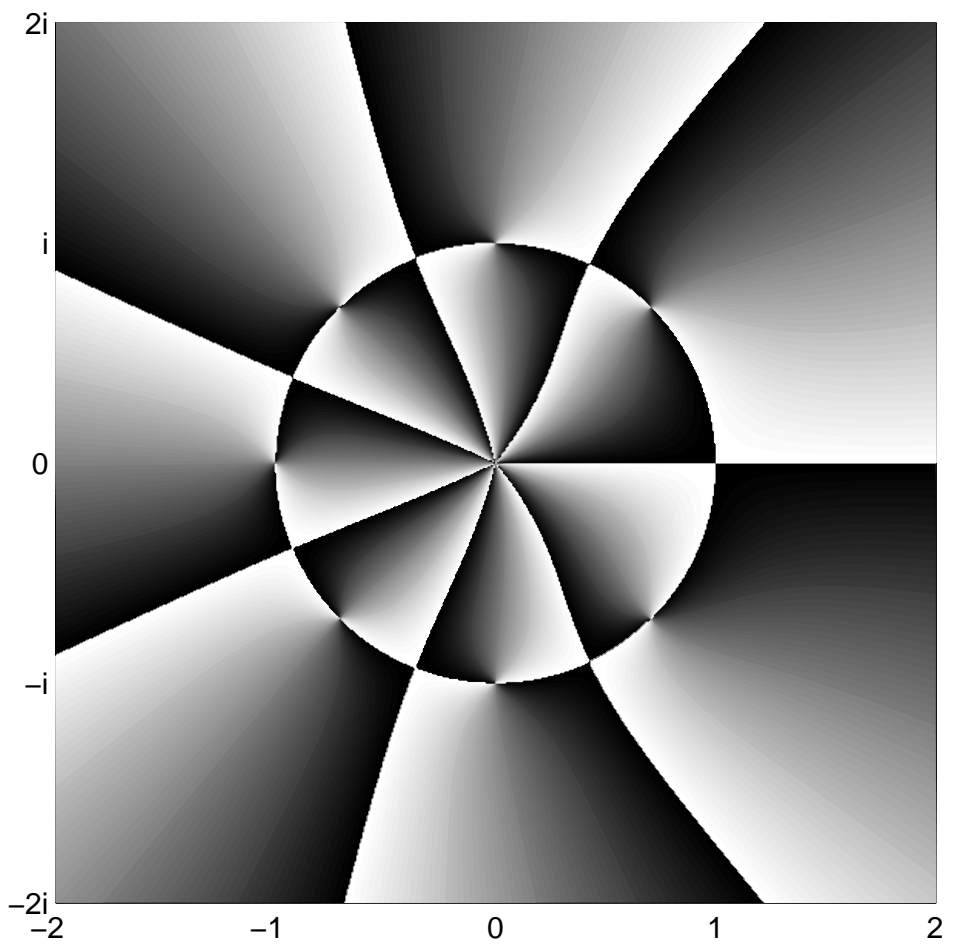

FIG. 2.2. This is a contour plot of $\arg \Phi(\zeta)$ with $N=7$ in the case $\sigma=1$. The zero contour is visible as the set of curves across which the color jumps from black to white.

In view of these symmetries and the fact that the spectral radius depends on the magnitude of the eigenvalues, we may assume, without loss of generality, that $|\zeta| \geq 1$ and $\operatorname{Im} \zeta \geq 0$. It is easily verified that

$$
\Phi\left(e^{z}\right)=\sigma \frac{\sinh ^{2} \frac{z}{2}}{\sinh ^{2} \frac{(N+1) z}{2}} \quad \text { and } \quad \Psi\left(e^{z}\right)=\tau \frac{\sinh ^{2} \frac{N z}{2}}{\sinh ^{2} \frac{(N+1) z}{2}}
$$

In light of these observations we transform system (1.10) into a system of equations in two real variables as follows. Let $D=\left\{1,2, \ldots\left\lfloor\frac{N+1}{2}\right\rfloor\right\}$, and let $P=\frac{2 \pi}{N+1} D$, and let $\mathcal{U}=[0, \infty) \times[0, \pi] \subset \mathbf{R}^{2}$. Define the functions $h, f, g: \mathcal{U} \backslash(\{0\} \times P) \rightarrow \mathbf{R}$ by

$$
\begin{aligned}
& h(x, y)=\arg \Phi\left(e^{x+i y}\right), \\
& f(x, y)=\left|\Phi\left(e^{x+i y}\right)\right|^{2}, \\
& g(x, y)=\left|\Psi\left(e^{x+i y}\right)\right|^{2} .
\end{aligned}
$$

The following lemmas provide explicit expressions for the functions $f, g$ and $h$. 


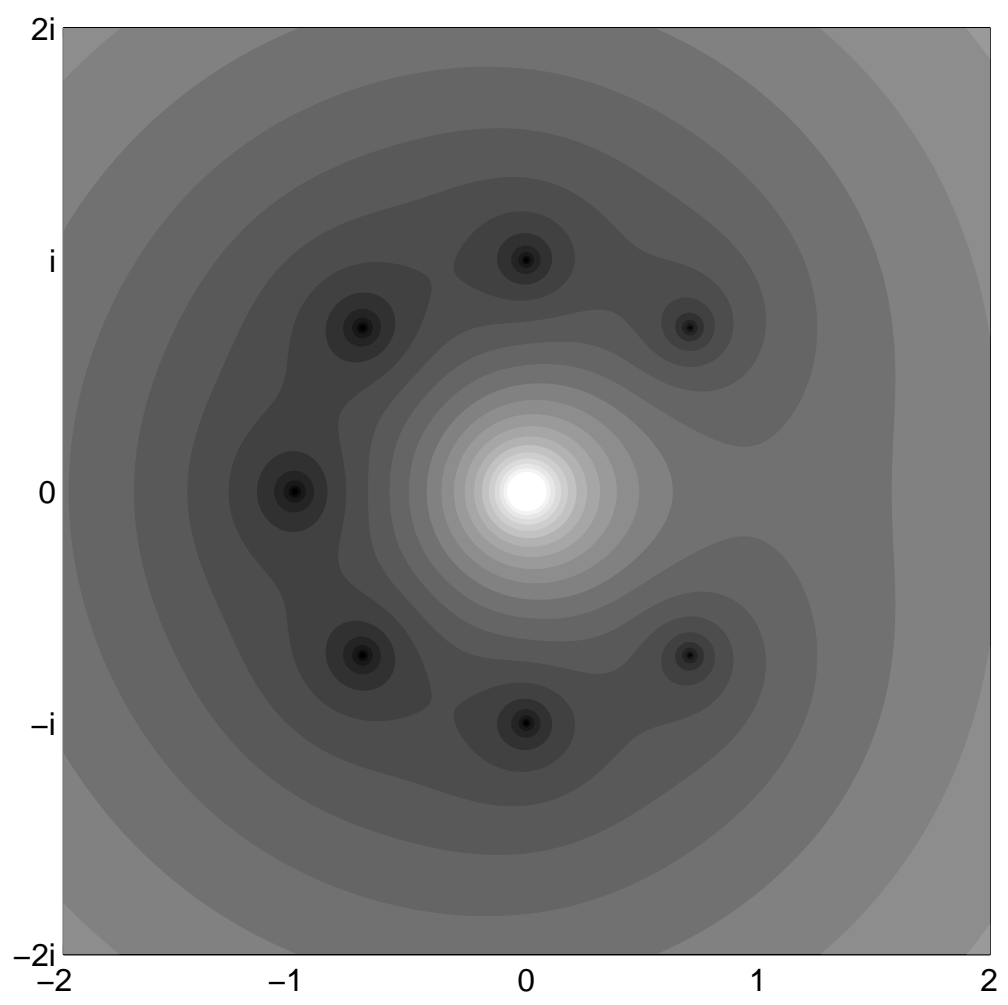

FIG. 2.3. This is a contour plot of $|\Phi(\zeta)|$ with $N=7$.

LEMma 2.1. We have

$$
f(x, y)=\left\{\begin{array}{ccc}
\frac{1}{(N+1)^{2}} & : \quad x=y=0, \\
\frac{\cosh x-\cos y}{\cosh ((N+1) x)-\cos ((N+1) y)} & : & \text { otherwise }
\end{array}\right.
$$

and

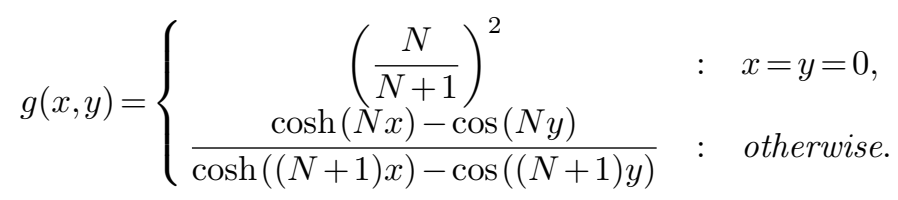

LEMMA 2.2. In the case $\sigma=-1$, we have $h(x, y)=0$ if and only if $x>0$ and either,

$$
\text { (i) } \tanh \frac{(N+1) x}{2} \tanh \frac{x}{2}+\tan \frac{(N+1) y}{2} \tan \frac{y}{2}=0
$$

or (ii) $N$ is odd and $y=\pi$. In the case $\sigma=1$, we have $h(x, y)=0$ if and only if either,

$$
\text { (i) } \tanh \frac{(N+1) x}{2} \tan \frac{y}{2}-\tan \frac{(N+1) y}{2} \tanh \frac{x}{2}=0
$$




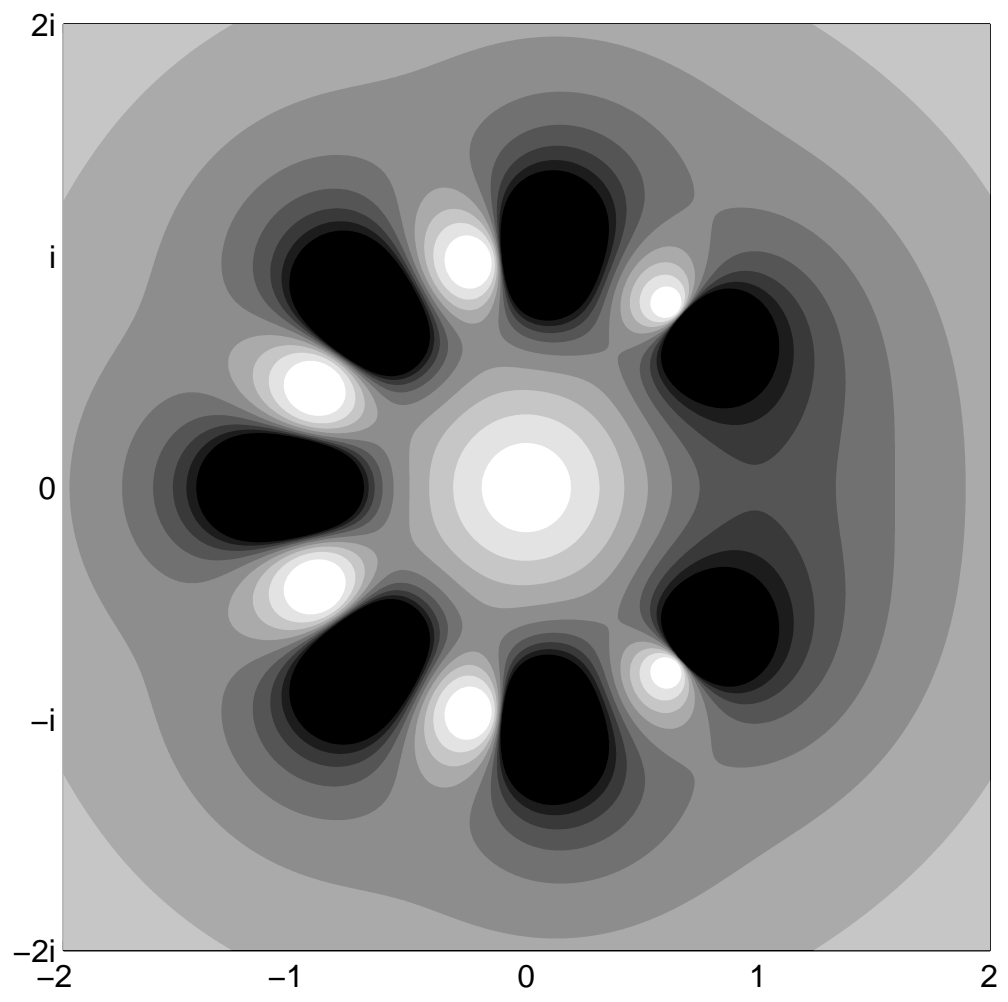

FIG. 2.4. This is a contour plot of $|\Psi(\zeta)|$ with $N=7$.

or (ii) $N$ is even and $y=\pi$.

\section{Proof of Lemma 2.1.}

Proof. From (2.11) we have

$$
\begin{aligned}
f(x, y) & =\frac{\sinh \frac{x+i y}{2} \sinh \frac{x-i y}{2}}{\sinh \frac{(N+1)(x+i y)}{2} \sinh \frac{(N+1)(x-i y)}{2}} \\
= & \frac{\sinh ^{2} \frac{x}{2} \cos ^{2} \frac{y}{2}+\cosh ^{2} \frac{x}{2} \sin ^{2} \frac{y}{2}}{\sinh ^{2} \frac{(N+1) x}{2} \cos ^{2} \frac{(N+1) y}{2}+\cosh ^{2} \frac{(N+1) x}{2} \sin ^{2} \frac{(N+1) y}{2}}
\end{aligned}
$$

and

$$
\begin{aligned}
g(x, y) & =\frac{\sinh \frac{N(x+i y)}{2} \sinh \frac{N(x-i y)}{2}}{\sinh \frac{(N+1)(x+i y)}{2} \sinh \frac{(N+1)(x-i y)}{2}} \\
= & \frac{\sinh ^{2} \frac{N x}{2} \cos ^{2} \frac{N y}{2}+\cosh ^{2} \frac{N x}{2} \sin ^{2} \frac{N y}{2}}{\sinh ^{2} \frac{(N+1) x}{2} \cos ^{2} \frac{(N+1) y}{2}+\cosh ^{2} \frac{(N+1) x}{2} \sin ^{2} \frac{(N+1) y}{2}} .
\end{aligned}
$$


These simplify by half-angle identities to

$$
f(x, y)=\frac{T(x, y)}{T((N+1) x,(N+1) y)}
$$

and

$$
g(x, y)=\frac{T(N x, N y)}{T((N+1) x,(N+1) y)},
$$

where

$$
\begin{aligned}
T(x, y) & =(\cosh x-1)(1+\cos y)+(\cosh x+1)(1-\cos y) \\
& =2(\cosh x-\cos y) .
\end{aligned}
$$

Proof of Lemma 2.2.

Proof. We prove the lemma for the case $\sigma=-1$. It follows from equation (2.11) that $h(x, y)=0$ if and only if

$$
\operatorname{Re}\left[\frac{\sinh \frac{x+i y}{2}}{\sinh \frac{(N+1)(x+i y)}{2}}\right]=0 .
$$

In order to simplify (2.15) we multiply the expression inside brackets by $A / A$, where $A=\sinh \frac{(N+1)(x-i y)}{2}$. We then expand the hyperbolic sine terms using angle-sum identities and simplify to obtain

$$
\begin{aligned}
\sinh \frac{x}{2} \cos \frac{y}{2} \sinh \frac{(N+1) x}{2} \cos \frac{(N+1) y}{2} & \\
& +\cosh \frac{x}{2} \sin \frac{y}{2} \cosh \frac{(N+1) x}{2} \sin \frac{(N+1) y}{2}=0 .
\end{aligned}
$$

We consider the two subcases (a) $y<\pi$ and (b) $y=\pi$. In subcase (a), each factor of (2.16) is positive when $x>0$ except possibly the two factors $\cos \frac{(N+1) y}{2}$ and $\sin \frac{(N+1) y}{2}$ which must therefore have opposite signs. We divide $(2.16)$ by the quantity, $\cos \frac{y}{2} \cos \frac{(N+1) y}{2} \cosh \frac{x}{2} \cosh \frac{(N+1) x}{2}$, which is non-zero, to obtain (2.14a). In subcase (b), the first term of (2.16) vanishes; the second term vanishes for $x>0$ if and only if $N$ is odd.

We omit the proof for the case $\sigma=1$ as it proceeds in the same manner as the case $\sigma=-1$.

We have seen that by mapping the $\zeta$-plane onto the real plane, the transformed functions $f, g$ and $h$ have a reduced interdependence among their real variables. The corresponding transformation of system 1.10 is,

$$
\begin{aligned}
0 & =h(x, y), \\
b^{2} & =\frac{\cosh x-\cos y}{\cosh ((N+1) x)-\cos ((N+1) y)}, \\
l^{2} & =\frac{\cosh (N x)-\cos (N y)}{\cosh ((N+1) x)-\cos ((N+1) y)} .
\end{aligned}
$$


This follows from Lemma 2.1. (Note that we have not written out an equivalent expression for $h(x, y)$ here because, by Lemma 2.2 , it is not easily written as a single expression.)

\section{The case $\sigma=-1$}

In order to prove Theorem 1.4 for $\sigma=-1$ we need the result of the following theorem which identifies the particular solution of system (2.17) that corresponds to the largest eigenvalue of $S(b)$. The proof of Theorem 3.1 follows the proof of Theorem 1.4 in this section.

TheOREm 3.1. Let $N$ be a positive integer and define the intervals $J, K \subset \mathbf{R}$ as

$$
J=\left(\frac{1}{N+1}, \infty\right) \text { and } K=N J .
$$

Let $D=\left\{1,2, \ldots,\left\lfloor\frac{N+1}{2}\right\rfloor\right\}$ and for each $k \in D$ define the subset $I_{k} \subset \mathbf{R}$ as

$$
I_{k}=\left[\frac{(2 k-1) \pi}{N}, \frac{2 k \pi}{N+1}\right] .
$$

For each $k \in D$ there exist functions $x_{k}, z_{k} \in C_{\infty}(J, \mathbf{R})$ and $y_{k} \in C_{\infty}\left(J, I_{k}\right)$ such that for each $b \in J$ system (2.17) has exactly the one solution, $(x, y, b, l)=$ $\left(x_{k}(b), y_{k}(b), b, z_{k}(b)\right)$, satisfying $x>0$ and $y \in I_{k}$. Each $z_{k}(b)$ is strictly increasing on $J$. Further, for each $i, j \in D$ such that $i<j$ we have $z_{i}(b)>z_{j}(b)$.

For each $k \in D$ there exist functions $\tilde{x}_{k}, \tilde{z}_{k} \in C_{\infty}(K, \mathbf{R})$ and $\tilde{y}_{k} \in C_{\infty}\left(K, I_{k}\right)$ such that for each $l \in K$ system (2.17) has exactly the one solution, $(x, y, b, l)=$ $\left(\tilde{x}_{k}(l), \tilde{y}_{k}(l), \tilde{z}_{k}(l), l\right)$, satisfying $x>0$ and $y \in I_{k}$. Further, for each $i, j \in D$ such that $i<j$ we have $\tilde{z}_{i}(l)<\tilde{z}_{j}(l)$.

Proof of Theorem 1.4, Case $\sigma=-1$.

Proof. Assume that Theorem 1.4 is true for $\sigma=1$. Choose $b$ such that $0<b \leq$ $1 /(N+1)$. The matrices $S(b ; \sigma=-1 ; \tau=1)$ and $S(b ; \sigma=1 ; \tau=1)$ differ only in the signs of corresponding elements. Since the latter matrix is non-negative, its spectral radius is an upper bound for the spectral radius of the former matrix. It follows from Theorem 1.4 that $\operatorname{spr} S(b ; \sigma=-1 ; \tau=1)<1$. By Lemma 1.5 the spectral radius of $S(b)$ is independent of $\tau$. We conclude that $\operatorname{spr} S(b ; \sigma=-1)<1$. Since $b \leq 1 /(N+1)$ was chosen arbitrarily we conclude that $b_{*}>1 /(N+1)$.

Let $\left(x_{1}, y_{1}, b_{1}, l_{1}\right)$ be the unique solution to (2.17) satisfying $x_{1}>0, y_{1} \in I_{1}$ and $l_{1}=1$. Such a solution exists by the second part of Theorem 3.1 and is given by $x_{1}=\tilde{x}_{1}(1), y_{1}=\tilde{y}_{1}(1)$, and $b_{1}=\tilde{z}_{1}(1)$. Therefore $b_{1} \geq b_{*}>1 /(N+1)$ by definition of $b_{*}$. It follows from the first part of Theorem 3.1 that $z_{1}\left(b_{1}\right)=1$ and that for each $b>1 /(N+1)$ the spectral radius of $S(b)$ is $z_{1}(b)$, which is strictly increasing. This proves that $b_{*}=b_{1}$. We postpone the computation of $b_{1}$ until Section 4 .

In order to prove Theorem 3.1 we will need the following Lemmas 3.2-3.6. We postpone the proofs of these technical lemmas until the Appendix.

Lemma 3.2. Let $D$ and $I_{k}$ be defined as in Theorem 3.1. There exist functions $\left\{\gamma_{k}\right\}_{k \in D} \subset C_{\infty}\left(\mathbf{R}_{\geq 0}, I_{k}\right)$ such that $h(x, y)=0$ if and only if $y=\gamma_{k}(x)$ for some $k \in D$ and $x \geq 0$. Each $\gamma_{k}$ is decreasing.

The next lemma expresses equation (2.17a) in terms of the same trigonometric and hyperbolic-trigonometric functions of $x$ and $y$ that appear in equation (2.17b). 
Lemma 3.3. Suppose $h(x, y)=0$. Then

$$
(\sigma s-t)(1-\sigma u v)+(\sigma s t-1)(\sigma u-v)=0,
$$

where $s=\cosh x, t=\cosh ((N+1) x), u=\cos y$, and $v=\cos ((N+1) y)$. Suppose in addition that $x>0$. Then

$$
\sigma \sin ((N+1) y) \geq 0
$$

We have stated this in terms of $\sigma$ because we will need this result in the treatment of the case $\sigma=1$ as well.

LEMma 3.4. Let $b>0$ and $l>0$ be real and let $(x, y) \in \mathbf{R}_{>0} \times[0, \pi]$ such that $h(x, y)=0$. Then (i) $f(x, y)=b^{2}$ if and only if

$$
u(t-\sigma s)-s t+\sigma+b^{2}\left(t^{2}-1\right)=0,
$$

and (ii) $g(x, y)=l^{2} \quad$ if and only if

$$
(u+\sigma t)(\cosh (N x)-\sigma)-\sigma l^{2}\left(t^{2}-1\right)=0,
$$

where $s, t, u$, and $v$ are as in Lemma 3.3.

Lemma 3.4 states that we can eliminate the quantity $v=\cos ((N+1) y)$ from system 2.17. After eliminating $v$, the only $y$ dependence of system 2.17 is through $u=\cos y$. We solve each of the equations (3.3) and (3.4) for $u$ to obtain two functions, $\phi$ and $\psi$, defined as follows.

$$
\begin{aligned}
& u=\phi(x, b) \equiv \frac{s t+1-b^{2}\left(t^{2}-1\right)}{s+t}, \\
& u=\psi(x, l) \equiv t-l^{2} \frac{t^{2}-1}{\cosh (N x)+1} .
\end{aligned}
$$

Thus, as a result of Lemma 3.4, the system (2.17) is equivalent to the system,

$$
\begin{aligned}
& h(x, y)=0, \\
& \phi(x, b)=\cos y, \\
& \psi(x, l)=\cos y .
\end{aligned}
$$

We will need the following facts about functions $\phi$ and $\psi$.

LEMma 3.5. Let $N$ be a positive integer and let $b>\frac{1}{N+1}$. Then $\phi_{x}(x, b)<0$ for each $x>0$.

Lemma 3.6. Let $N$ be a positive integer and let $l>\frac{N}{N+1}$. Then $\psi_{x}(x, l)<0$ for each $x>0$ such that $\psi(x, l)<1$.

Assuming lemmas 3.2-3.6, we can now give the

Proof of Theorem 3.1.

Proof. Define the function $H: \mathbf{R}_{>0} \times J \times K \rightarrow \mathbf{R}$ by

$$
H(x, b, l)=\frac{s+t}{t^{2}-1}(\phi(x, b)-\psi(x, l)) .
$$


We need the following fact about $H$.

Claim 3.1. $H_{x}(x, b, l)>0$.

Choose $b$ such that $b>1 /(N+1)$. We have $\phi(0, b)=1$ and $\phi(x, b) \rightarrow-\infty$ as $x \rightarrow \infty$. It follows from Lemma 3.5 that the curve define by, $\phi(x, b)=\cos y$, crosses each curve $y=\gamma_{k}(x)$ of Lemma 3.2 exactly once. Label these intersection points $\left(u_{k}, v_{k}\right)$ such that $v_{k}=\gamma_{k}\left(u_{k}\right)$. Let $k \in D$ let $l_{k}=\sqrt{g\left(u_{k}, v_{k}\right)}$. By Lemma $3.4(x, y, b, l)=\left(u_{k}, v_{k}, b, l_{k}\right)$ is a solution to (3.5). We apply the Implicit Function Theorem to the $\mathrm{C}_{\infty}$ function

$$
F(x, y, b, l)=\left(y-\gamma_{k}(x), \cos y-\phi(x, b), H(x, b, l)\right) .
$$

The determinant of the Jacobian of $\mathrm{F}$ with respect to variables $x, y$ and $l$ is

$$
\begin{aligned}
\left|\frac{\partial F(x, y, b, l)}{\partial(x, y, l)}\right| & =\left|\begin{array}{ccc}
-\gamma_{k}^{\prime}(x) & 1 & 0 \\
-\phi_{x}(x, b) & -\sin y & 0 \\
H_{x}(x, b, l) & 0 & H_{l}(x, b, l)
\end{array}\right| \\
& =\left(\gamma_{k}^{\prime}(x) \sin y+\phi_{x}(x, b)\right) H_{l}(x, b, l) .
\end{aligned}
$$

The right-hand side of (3.6) is negative at $(x, y, b, l)=\left(u_{k}, v_{k}, b, l_{k}\right)$ and $F\left(u_{k}, v_{k}, b, l_{k}\right)=0$. By the Implicit Function Theorem there exists an open neighborhood $U \subset \mathbf{R}$ of $b$ and functions $x_{k}, y_{k}, z_{k} \in \mathrm{C}_{\infty}(U, \mathbf{R})$ such that $F\left(x_{k}(b), y_{k}(b), b, z_{k}(b)\right)=0$ for each $b \in U$. Because the choice of $b$ was arbitrary, we can take $U=J$.

We will now prove that $z_{k}^{\prime}>0$ on $J$. Let $k \in D$ and let $(x, y, b, l)$ be a solution to (2.17) such that $x>0, y \in I_{k}$ and $b \in J$. Then $x=x_{k}(b), y=y_{k}(b)$ and $l=z_{k}(b)$. By Lemma 3.2 it follows that $y=\gamma_{k}(x)$ and by Lemma 3.4 it follows that

$$
\cos \gamma_{k}\left(x_{k}(b)\right)-\phi\left(x_{k}(b), b\right)=0 .
$$

Differentiating (3.7) with respect to $b$ we find that

$$
\frac{\mathrm{d} x_{k}}{\mathrm{~d} b}=-\frac{\phi_{b}\left(x_{k}(b), b\right)}{\phi_{x}\left(x_{k}(b), b\right)+\gamma_{k}^{\prime}\left(x_{k}(b)\right) \sin \gamma_{k}\left(x_{k}(b)\right)}<0 .
$$

From Lemma 3.4 it follows that, $H\left(x_{k}(b), b, z_{k}(b)\right)=0$. Differentiating this with respect to $b$ we find that

$$
\frac{\mathrm{d} z_{k}}{\mathrm{~d} b}=-\frac{\frac{\mathrm{d} x_{k}}{\mathrm{~d} b} H_{x}\left(x_{k}(b), b, z_{k}(b)\right)+H_{b}\left(x_{k}(b), b, z_{k}(b)\right)}{H_{l}\left(x_{k}(b), b, z_{k}(b)\right)}
$$

Since $H_{b}(x, b, l)<0$ and $H_{l}(x, b, l)>0$ it follows from Claim 3.1 that $z_{k}^{\prime}(b)>0$.

Suppose that $i, j \in D$ such that $i<j$. Along the curve $H(x, b, l)=0, b$ fixed, we have

$$
\frac{\mathrm{d} l}{\mathrm{~d} x}=-\frac{H_{x}(x, b, l)}{H_{l}(x, b, l)} .
$$

It follows from Claim 3.1 that $\frac{\mathrm{d} l}{\mathrm{~d} x}<0$. Differentiating equation $(3.5 \mathrm{~b})$ with respect to $y$ while holding $b$ fixed we find

$$
\frac{\mathrm{d} x}{\mathrm{~d} y}=-\frac{\sin y}{\phi_{x}(x, b)}
$$


By Lemma 3.5, $\frac{\mathrm{d} x}{\mathrm{~d} y}>0$. We therefore have $y_{i}(b)<y_{j}(b)$ which implies that $x_{i}(b)<x_{j}(b)$ which implies that $l_{i} \equiv z_{i}(b)>z_{j}(b) \equiv l_{j}$. This proves the first part of the theorem.

The proof of the second part of the theorem proceeds in the same manner and is omitted.

Proof of Claim 3.1.

Proof. We must show that $H_{x}(x, b, l)>0$ whenever $x>0, b>0$ and $l>0$. We have

$$
\begin{aligned}
H(x, b, l) & =\frac{s+t}{t^{2}-1}(\phi(x, b)-\psi(x, l)) \\
& =\frac{s+t}{t^{2}-1}\left(\frac{s t+1-b^{2}\left(t^{2}-1\right)}{s+t}-t+l^{2} \frac{t^{2}-1}{\cosh (N x)+1}\right) \\
& =-1-b^{2}+l^{2} \frac{s+t}{\cosh (N x)+1} \\
& =-1-b^{2}+l^{2} \frac{\cosh x+\cosh ((N+1) x)}{\cosh (N x)+1}
\end{aligned}
$$

The function

$$
\begin{aligned}
\frac{\cosh x+\cosh ((N+1) x)}{\cosh (N x)+1} & =\frac{\cosh x+\cosh (N x) \cosh x+\sinh (N x) \sinh x}{\cosh (N x)+1} \\
& =\cosh x+\frac{2 \sinh \frac{N x}{2} \cosh \frac{N x}{2} \sinh x}{2 \cosh ^{2} \frac{N x}{2}} \\
& =\cosh x+\tanh \frac{N x}{2} \sinh x,
\end{aligned}
$$

is increasing on $x>0$. This proves the claim.

\section{Asymptotics}

In this section we compute the value of $b_{*}$ in the case $\sigma=-1$ where $b_{*}=b_{1}$ and $b_{1}$ is defined as in the proof of Theorem 1.4 in Section 3.

Let $\left(x_{1}, y_{1}\right)$ be the intersection point of the curve $y=\gamma_{1}(x)$, as defined in Lemma 3.2 , with the curve defined implicitly by the equation $\cos y=\psi(x, 1)$. We want to expand $x_{1}$ and $y_{1}$ in powers of $N$. We know from Lemma 3.2 that $y$ is of order $N^{-1}$ along $\gamma_{1}$, hence $y_{1}=\mathrm{O}\left(N^{-1}\right)$. We determine the asymptotic expansions of $x_{1}$ and $y_{1}$ as follows. We expand $x_{1}$ and $y_{1}$ as

$$
\begin{aligned}
& x_{1}=a_{1} N^{-\alpha}+a_{2} N^{-\alpha-\delta}+h . o . t . \\
& y_{1}=\frac{2 \pi}{N+1}+b_{1} N^{-1-\epsilon}+\text { h.o.t. }
\end{aligned}
$$

We first determine the exponents $\alpha, \delta$, and $\epsilon$. We have

$$
\begin{aligned}
\cos y_{1} & =\psi\left(x_{1}, 1\right) \\
& =\cosh \left((N+1) x_{1}\right)-\frac{\cosh ^{2}\left((N+1) x_{1}\right)-1}{\cosh \left(N x_{1}\right)+1} .
\end{aligned}
$$

Subtract 1 from each side of (4.3), multiply through by, $\cosh \left(N x_{1}\right)+1$, and factor as

$$
\begin{aligned}
& \left(\cosh \left(N x_{1}\right)+1\right)\left(\cos y_{1}-1\right) \\
= & \left(\cosh \left((N+1) x_{1}\right)-1\right)\left(\cosh \left(N x_{1}\right)-\cosh \left((N+1) x_{1}\right)\right) \\
= & 2\left(\cosh \left((N+1) x_{1}\right)-1\right) \sinh \frac{(2 N+1) x_{1}}{2} \sinh \frac{x_{1}}{2} .
\end{aligned}
$$


We substitute for $x_{1}$ and $y_{1}$ from (4.1) and (4.2). In order for our expansions to be valid we assume that $\alpha>1$. We have

$$
\begin{aligned}
\cosh \left(N x_{1}\right)+1 & =2+c_{1} N^{2-2 \alpha}+\text { h.o.t., } \\
\cos y_{1}-1 & =c_{2} N^{-2}+c_{3} N^{-2-\epsilon}+\text { h.o.t. }, \\
\cosh \left((N+1) x_{1}\right)-1 & =c_{4} N^{2-2 \alpha}+c_{5} N^{2-2 \alpha-\delta}+\text { h.o.t. }, \\
\sinh \frac{(2 N+1) x_{1}}{2} & =c_{6} N^{1-\alpha}+c_{7} N^{1-\alpha-\delta}+\text { h.o.t, } \\
\sinh \frac{x_{1}}{2} & =c_{8} N^{-\alpha}+c_{9} N^{-\alpha-\delta}+\text { h.o.t., }
\end{aligned}
$$

where the $c_{i}$ are constants that depend on the $a_{i}$ and $b_{i}$. Substitute these into (4.4) to find

$$
\begin{aligned}
2 c_{2} N^{-2}+c_{1} c_{2} N^{-2 \alpha}+2 c_{3} N^{-2-\epsilon}+\text { h.o.t } \\
\quad=2 c_{4} c_{6} c_{8} N^{3-4 \alpha}+2\left(c_{4} c_{7} c_{8}+c_{5} c_{6} c_{8}+c_{4} c_{6} c_{9}\right) N^{3-4 \alpha-\delta}+\text { h.o.t. }
\end{aligned}
$$

Matching the leading order terms on both sides of (4.5) we find that

$$
\alpha=\frac{5}{4} \text {. }
$$

Equation (4.5) does not uniquely determine $\delta$ and $\epsilon$. At this point we could substitute $x_{1}$ and $y_{1}$ into the equation $h(x, y)=0$, but we find that it is much simpler to guess that $2 \alpha=2+\epsilon$. Then

$$
\epsilon=\frac{1}{2}
$$

At the end we show that this correct. Matching second-order terms on both sides of (4.5) we find that

$$
-2 \alpha=3-4 \alpha-\delta
$$

which implies that

$$
\delta=\frac{1}{2}
$$

We are thus led to expand $x_{1}$ and $y_{1}$ as follows.

$$
\begin{aligned}
& x_{1}=a_{1} N^{-5 / 4}+a_{2} N^{-7 / 4}+a_{3} N^{-9 / 4}+\ldots, \\
& y_{1}=\frac{2 \pi}{N}+b_{1} N^{-3 / 2}+b_{2} N^{-2}+\ldots
\end{aligned}
$$

We determine the $a_{i}$ and $b_{i}$ in expansions (4.6) and (4.7). The point $\left(x_{1}, y_{1}\right)$ is the intersection point of the curves $y=\gamma_{1}(x)$ and $\cos y=\psi(x, 1)$. Thus

$$
\begin{aligned}
& \left(\cosh x_{1} \cosh \left((N+1) x_{1}\right)+1\right)\left(\cos y_{1}+\cos \left((N+1) y_{1}\right)\right) \\
& \quad-\left(\cosh x_{1}+\cosh \left((N+1) x_{1}\right)\right)\left(1+\cos y_{1}+\cos \left((N+1) y_{1}\right)\right)=0
\end{aligned}
$$

and

$$
\begin{aligned}
& \left(\cosh \left(N x_{1}\right)+1\right)\left(\cos y_{1}-1\right) \\
= & 2\left(\cosh \left((N+1) x_{1}\right)-1\right) \sinh \frac{(2 N+1) x_{1}}{2} \sinh \frac{x_{1}}{2} .
\end{aligned}
$$


Equation (4.8) is obtained from equation (3.1) by setting $\sigma=-1$ and equation (4.9) is a reiteration of (4.4). We substitute $x_{1}$ and $y_{1}$ from (4.6) and (4.7), respectively, into (4.8) and (4.9) and expand in powers of $N$. By matching coefficients of powers of $N$ on both sides of these equations we are led to algebraic expressions involving the coefficients $a_{i}$ and $b_{i}$ which we solve to determine the $a_{i}$ and $b_{i}$. Rather than write these equations out in laborious detail, we will state the results obtained with Mathematica.

$$
\begin{aligned}
& x_{1}=2^{3 / 4} \pi^{1 / 2} N^{-5 / 4}-2^{-3 / 4} \pi^{-1 / 2} N^{-7 / 4}+\mathrm{O}\left(N^{-9 / 4}\right), \\
& y_{1}=2 \pi N^{-1}-2^{1 / 2} N^{-3 / 2}-\frac{5 \pi}{3} N^{-2}+\mathrm{O}\left(N^{-5 / 2}\right) .
\end{aligned}
$$

Because $x_{1}$ and $y_{1}$ have unique asymptotic expansions of the form (4.6) and (4.7), respectively, and these expansions are given by (4.10) and (4.11), respectively, our guess that $2 \alpha=2+\epsilon$ is correct. That is to say, if this guess had been incorrect we would have been led to an algebraic inconsistency in the relations between the $a_{i}$ and $b_{i}$.

We now use the equation $b^{2}=f(x, y)$ to determine the threshold value of $b$. From equation (2.12) we have

$$
b^{2}=\frac{\cosh x-\cos y}{\cosh ((N+1) x)-\cos ((N+1) y)} .
$$

Substituting $x_{1}$ and $y_{1}$ from (4.10) and (4.11), respectively, into (4.12) we obtain

$$
b_{*}^{2}=2^{1 / 2} \pi N^{-3 / 2}-\left(\frac{\pi^{2}}{3}+1\right) N^{-2}+\mathrm{O}\left(N^{-5 / 2}\right)
$$

and

$$
b_{*}=2^{1 / 4} \pi^{1 / 2} N^{-3 / 4}+\mathrm{O}\left(N^{-5 / 4}\right) .
$$

We note here that the expansion obtained for $b_{*}$ is valid when $x_{1}=\mathrm{o}\left(N^{-1}\right)$. Herein lies the difficulty in approximating the points $\left(x_{k}, y_{k}\right)$ for $k>1$. When $k$ is of the order $N^{1 / 2}$, we have,

$$
y_{k}=\mathrm{O}\left(\frac{2 k \pi}{N+1}\right)=\mathrm{O}\left(N^{-1 / 2}\right)
$$

Consequently, we obtain from equation (4.4) that $x=\mathrm{O}\left(N^{-1}\right)$. The Taylor series for $\cosh ((N+1) x)$ converges very slowly when $x=\mathrm{O}\left(N^{-1}\right)$ so that the corresponding expansion for $b_{*}$, if convergent, will converge slowly. With the result of Theorem 3.1 we avoid this difficulty.

\section{The case $\sigma=1$}

In this section we prove Theorem 1.4 when $\sigma=1$. We need the following lemma whose proof we postpone until the Appendix.

Lemma 5.1. Let $N$ be an integer, $N \geq 2$. Let $D$ be defined as in Theorem 3.1. For each $j \in D$ let $p_{j}=\frac{2 \pi j}{N+1}$. Let $P=\left\{p_{j}\right\}_{j \in D}$. Define the functions $\phi, \psi:[0, \pi] \backslash P \rightarrow \mathbf{R}$ by

$$
\phi(y)=f(0, y) \quad \text { and } \quad \psi(y)=g(0, y) .
$$


Each function $\phi$ and $\psi$ is strictly increasing on $\left[0, p_{1}\right)$ and strictly convex on each interval $\left(p_{j-1}, p_{j}\right)$ for each $j \in D$.

$$
\text { Let } y_{1}=\frac{2 \pi}{2 N+1} \text {. Then } \phi(y)>\phi\left(y_{1}\right) \text { on }\left(y_{1}, \pi\right] \text {. }
$$

Proof of Theorem 1.4, Case $\sigma=1$.

Proof. Let $\phi, \psi, y_{1}$ and $P$ be defined as in the previous lemma and let

$$
b_{1}=\sqrt{\phi\left(y_{1}\right)}=\frac{\sin \frac{y_{1}}{2}}{\sin \frac{(N+1) y_{1}}{2}}=\frac{\sin \frac{y_{1}}{2}}{\cos \frac{y_{1}}{4}}=2 \sin \frac{y_{1}}{4} \text {. }
$$

Then

$$
\psi\left(y_{1}\right)=\frac{\sin ^{2} \frac{N y_{1}}{2}}{\sin ^{2} \frac{(N+1) y_{1}}{2}}=\frac{\cos ^{2} \frac{\pi}{2(2 N+1)}}{\cos ^{2} \frac{\pi}{2(2 N+1)}}=1 .
$$

Hence $S\left(b_{1}\right)$ has an eigenvalue of modulus 1 and we must have $b_{*} \leq b_{1}$. Suppose $b_{*}<b_{1}$. Choose $b$ such that $b_{*}<b<b_{1}$. Then there exist $x^{\prime} \geq 0$ and $y^{\prime} \in[0, \pi] \backslash P$ such that

$$
f\left(x^{\prime}, y^{\prime}\right)=b^{2} \quad \text { and } \quad g\left(x^{\prime}, y^{\prime}\right) \geq 1 .
$$

If $x^{\prime}>0$ then Lemma 3.4 implies that

$$
\begin{aligned}
1 \leq g\left(x^{\prime}, y^{\prime}\right) & =\frac{\left(\cos y^{\prime}+\cosh \left((N+1) x^{\prime}\right)\right)\left(\cosh \left(N x^{\prime}\right)-1\right)}{\cosh ^{2}\left((N+1) x^{\prime}\right)-1} \\
& <\frac{\cosh \left(N x^{\prime}\right)-1}{\cosh \left((N+1) x^{\prime}\right)-1}<1 .
\end{aligned}
$$

This is a contradiction, so we must have $x^{\prime}=0$. From Lemma 5.1 we first conclude that $y^{\prime} \in\left[0, y_{1}\right)$, and then conclude that

$$
g\left(0, y^{\prime}\right)=\psi\left(y^{\prime}\right)<\psi\left(y_{1}\right)=1 .
$$

This is a contradiction. We conclude that no such $x^{\prime}$ and $y^{\prime}$ exist and that therefore $b_{*}=b_{1}$. This proves the theorem.

Appendix A. In this section we prove Lemmas 3.2, 3.3, 3.4, 3.5, 3.6 and 5.1. Proof of Lemma 3.2.

Proof. By Lemma 2.2 we have in the case $\sigma=-1$ that, $h(x, y)=0$, if and only if either

$$
\text { (i) } \tanh \frac{(N+1) x}{2} \tanh \frac{x}{2}+\tan \frac{(N+1) y}{2} \tan \frac{y}{2}=0
$$

or (ii) $N$ is odd and $y=\pi$. The roots to (A.1) with $x=0$ are $y=\frac{2 k \pi}{N+1}$, $k \in D$. These points are not in the domain of $h(x, y)$. The first term maps $(0, \infty)$ onto $(0,1)$, thus the second term is in the range $(-1,0)$ at each root. The preimage of $(-1,0)$ under the second term is

$$
I_{k}=\left(\frac{(2 k-1) \pi}{N}, \frac{2 k \pi}{N+1}\right), \quad k=1, \ldots,\left\lfloor\frac{N}{2}\right\rfloor
$$


We see this as follows. We have

$$
\begin{aligned}
& \lim _{y \rightarrow \frac{(2 k-1) \pi}{N}+} \tan \frac{(N+1) y}{2} \tan \frac{y}{2} \\
= & \tan \left(\frac{(2 k-1) \pi}{2}+\frac{(2 k-1) \pi}{2 N}\right) \tan \frac{(2 k-1) \pi}{2 N} \\
= & -\cot \frac{(2 k-1) \pi}{2 N} \tan \frac{(2 k-1) \pi}{2 N} \\
= & -1
\end{aligned}
$$

and

$$
\left.\tan \frac{(N+1) y}{2} \tan \frac{y}{2}\right|_{y=\frac{2 k \pi}{N+1}}=0 .
$$

Because the second term is increasing on each $I_{k}$, the second term is a bijection from each $I_{k}$ to $(-1,0)$. Thus for each $x>0$, equation (A.1) has a unique solution $y_{k}(x)$ in each interval $I_{k}, k=1, \ldots,\left\lfloor\frac{N}{2}\right\rfloor$. Further, each $y_{k}(x)$ is a decreasing function of $x$. When $N$ is odd the equation $h(x, y)=0$ has the additional solution, $y=\pi$. This proves the lemma.

\section{Proof of Lemma 3.3.}

Proof. We prove the first part of the lemma for the case $\sigma=-1$. We have from (2.11) that, $h(x, y)=0$, if and only if

$$
\operatorname{Re}\left[\frac{\sinh \frac{x+i y}{2}}{\sinh \frac{(N+1)(x+i y)}{2}}\right]=0 .
$$

In order to simplify equation (A.2) we multiply the expression inside brackets by $A / A$ where $A=\sinh \frac{(N+1)(x-i y)}{2}$ and expanding with angle-sum identities to obtain

$$
\begin{aligned}
\sinh \frac{x}{2} \cos \frac{y}{2} \sinh \frac{(N+1) x}{2} \cos \frac{(N+1) y}{2} & \\
& +\cosh \frac{x}{2} \sin \frac{y}{2} \cosh \frac{(N+1) x}{2} \sin \frac{(N+1) y}{2}=0 .
\end{aligned}
$$

Equation (A.3) is of the form $P+Q=0$. Multiply (A.3) by $P-Q$ to obtain

$$
\begin{aligned}
\sinh ^{2} \frac{x}{2} \cos ^{2} \frac{y}{2} \sinh ^{2} \frac{(N+1) x}{2} & \cos ^{2} \frac{(N+1) y}{2} \\
& -\cosh ^{2} \frac{x}{2} \sin ^{2} \frac{y}{2} \cosh ^{2} \frac{(N+1) x}{2} \sin ^{2} \frac{(N+1) y}{2}=0 .
\end{aligned}
$$

Using the half-angle identities

$$
\begin{aligned}
\cosh ^{2} \frac{x}{2} & =\frac{\cosh x+1}{2}, & \sinh ^{2} \frac{x}{2} & =\frac{\cosh x-1}{2}, \\
\cos ^{2} \frac{y}{2} & =\frac{1+\cos y}{2}, & \sin ^{2} \frac{y}{2} & =\frac{1-\cos y}{2},
\end{aligned}
$$


(A.4) simplifies to

$$
\begin{aligned}
0 & =(s-1)(1+u)(t-1)(1+v)-(s+1)(1-u)(t+1)(1-v) \\
& =(t+s)(1+u v)-(s t+1)(u+v) .
\end{aligned}
$$

We prove the second part of the Lemma for the case $\sigma=-1$. Suppose that $x>0$ and $y$ satisfy the hypothesis of the Lemma. We treat the case $\sigma=-1$. It follows from (A.3) that either (i) $y<\pi$ or (ii) $y=\pi$ and $N$ is odd. In subcase (i) all factors in (A.3) are positive except possibly the two terms $\cos \frac{(N+1) y}{2}$ and $\sin \frac{(N+1) y}{2}$, which must therefore have opposite signs. Therefore

$$
\sigma \sin ((N+1) y)=2 \sigma \sin \frac{(N+1) y}{2} \cos \frac{(N+1) y}{2}>0 .
$$

In subcase (ii) we have $\sigma \sin ((N+1) y)=0$.

We omit the proof of the lemma for the case $\sigma=1$ as it proceeds in the same manner as the case $\sigma=-1$.

\section{Proof of Lemma 3.4.}

Proof. Suppose that $b, l, x$, and $y$ satisfy the hypotheses of the lemma. We prove (i). By Lemma 3.3 we have that (3.1) holds.

$$
(\sigma s-t)(1-\sigma u v)+(\sigma s t-1)(\sigma u-v)=0 .
$$

By Lemma 2.1 we have that $f(x, y)=b^{2}$ if and only if

$$
b^{2}=\frac{s-u}{t-v}
$$

We eliminate $v$ from (A.5) and (A.6) as follows. We solve (A.5) for $v$ to find

$$
v=\frac{\sigma s-t+(s t-\sigma) u}{\sigma(s t-\sigma+(\sigma s-t) u)} .
$$

Multiply equation (A.6) by, $t-v$, and substitute for $v$ from (A.7) to obtain

$$
b^{2}\left(t-\frac{\sigma s-t+(s t-\sigma) u}{\sigma(s t-\sigma+(\sigma s-t) u)}\right)=s-u .
$$

Multiply (A.8) by $\sigma(s t-\sigma+(\sigma s-t) u)$ and simplify to

$$
b^{2} \sigma(s-u)\left(t^{2}-1\right)=\sigma(s-u)(s t-\sigma+(\sigma s-t) u) .
$$

Divide (A.9) by $\sigma(s-u)$ to obtain (3.3). This proves (i).

We prove (ii). By Lemma 2.1 we have that $g(x, y)=l^{2}$ if and only if

$$
l^{2}=\frac{\cosh (N x)-\cos (N y)}{t-v} .
$$

We eliminate $v$ from (A.5) and (A.10) as follows. We rewrite (A.10) using angle-sum identities as

$$
l^{2}=\frac{s t-\sinh ((N+1) x) \sinh x-u v-\sin ((N+1) y) \sin y}{t-v} .
$$


By Lemma 3.3, part 2 we have that $\sigma \sin ((N+1) y)>0$ when $x>0$. Using this fact, equation (A.11) becomes

$$
l^{2}=\frac{s t-\sqrt{\left(t^{2}-1\right)\left(s^{2}-1\right)}-u v-\sigma \sqrt{\left(1-v^{2}\right)\left(1-u^{2}\right)}}{t-v} .
$$

We need the following identity for $h(x, y)=0$.

$$
\frac{(s-1)(t+\sigma)}{(s+1)(t-\sigma)}=\frac{(1-u)(1+\sigma v)}{(1+u)(1-\sigma v)} .
$$

We let $T=\sqrt{\left(t^{2}-1\right)\left(s^{2}-1\right)}$ and rewrite (A.12) as

$$
l^{2}(t-v)-s t+u v+\left(1+\sigma \frac{(1+u)(1-\sigma v)}{(s+1)(t-\sigma)}\right) T=0 .
$$

We solve (A.13) for quantities $v$ and $1-\sigma v$ to find

$$
\begin{aligned}
v & =\frac{\sigma s-t+(s t-\sigma) u}{\sigma(s t-\sigma+(\sigma s-t) u)}, \\
1-\sigma v & =\frac{(s+1)(t-\sigma)(1-u)}{s t-\sigma+(\sigma s-t) u} .
\end{aligned}
$$

Substituting these into (A.14) and simplifying we find

$$
\begin{aligned}
& (s t-T-\sigma) u^{2}+\left[(s t-T-\sigma)(\sigma t-s)-\sigma l^{2}\left(t^{2}-1\right)\right] u \\
& +\left(l^{2}-s\right)(\sigma s t-1) t+l^{2}(t-\sigma s)+\sigma s t T=0 .
\end{aligned}
$$

Equation (A.17) is of the form $A u^{2}+B u+C$ with coefficients $A, B$ and $C$ given by

$$
\begin{aligned}
& A=s t-T-\sigma=\cosh (N x)-\sigma, \\
& B=A(\sigma t-s)-\sigma l^{2}\left(t^{2}-1\right), \\
& C=l^{2}\left(\sigma s t^{2}-t-\sigma s+t\right)-\sigma s t(s t-T-\sigma)=\sigma l^{2} s\left(t^{2}-1\right)-\sigma A s t .
\end{aligned}
$$

The discriminant of (A.17) is

$$
B^{2}-4 A C=\left[A(t+\sigma s)-l^{2}\left(t^{2}-1\right)\right]^{2} .
$$

We restrict $x$ such that the quantity $A(t+\sigma s)-l^{2}\left(t^{2}-1\right)$ is non-negative. The solutions to (A.17) are

$$
\begin{aligned}
& u_{1}=-\sigma\left(t-\frac{l^{2}\left(t^{2}-1\right)}{A}\right), \\
& u_{2}=s .
\end{aligned}
$$

Recalling that $u=\cos y$ and $s=\cosh x$, the equation $u=u_{2}$, where $u_{2}$ is given by (A.20), has no solutions with $x>0$. Thus all solutions are given by $u_{1}$. The equation $u=u_{1}$ is equivalent to (3.4). This proves (ii).

\section{Proof of Lemma 3.5.}

Proof. Recall that

$$
\phi(x, b)=\frac{s t+1-b^{2}\left(t^{2}-1\right)}{s+t},
$$




$$
s=\cosh x \quad \text { and } \quad t=\cosh ((N+1) x) .
$$

Let $N$ be a positive integer and let $b>\frac{1}{N+1}$. We factor $\phi(x, b)-1$ as

$$
\phi(x, b)-1=\left(\frac{t-1}{t+s}\right)\left(s-1-b^{2}(t+1)\right) .
$$

The first factor on the right-hand side of equation (A.21) is positive and strictly increasing on $x>0$. Define the function

$$
g(x)=\cosh x-1-b^{2}(\cosh ((N+1) x)+1) .
$$

Differentiating $g$ twice we find

$$
\begin{aligned}
g^{\prime}(x) & =\sinh x-b^{2}(N+1) \sinh ((N+1) x), \\
g^{\prime \prime}(x) & =\cosh x-b^{2}(N+1)^{2} \cosh ((N+1) x) .
\end{aligned}
$$

We conclude that the function $\mathrm{g}$ is negative at the critical point $x=0$ and concave on $x>0$ and hence is negative and strictly decreasing on $x>0$. Therefore we have $\phi_{x}(x, b)<0$ on $x>0$.

\section{Proof of Lemma 3.6.}

Proof. Recall that

$$
\psi(x, l)=t-l^{2} \frac{t^{2}-1}{\cosh (N x)+1} \quad \text { and } \quad t=\cosh ((N+1) x) .
$$

Let $N$ be a positive integer and let $l>\frac{N}{N+1}$. We factor $\psi(x, l)-1$ as

$$
\psi(x, l)-1=\left(\frac{t-1}{\cosh (N x)+1}\right)\left(\cosh (N x)+1-l^{2}(t+1)\right) .
$$

The first factor on the right-hand side of equation (A.22) is positive and strictly increasing on $x>0$. Define the function

$$
g(x)=\cosh (N x)+1-l^{2}(\cosh ((N+1) x)+1) .
$$

Differentiating $g$ twice we find

$$
\begin{aligned}
g^{\prime}(x) & =N \sinh (N x)-l^{2}(N+1) \sinh ((N+1) x), \\
g^{\prime \prime}(x) & =N^{2} \cosh (N x)-l^{2}(N+1)^{2} \cosh ((N+1) x) .
\end{aligned}
$$

We conclude that the function $\mathrm{g}$ has a critical point at $x=0$ and is concave on $x>0$ and hence is strictly decreasing on $x>0$. Suppose $x>0$ such that $\psi(x, l)<1$. Then by equation $(\mathrm{A} .22)$ we must have $g(x)<0$. Therefore $\psi_{x}(x, b)<0$.

Proof of Lemma 5.1.

Proof. For each integer $k$ define the function $h_{k}:[0, \pi] \backslash P \rightarrow \mathbf{R}$ to be

$$
h_{k}(y)= \begin{cases}\frac{\sin \frac{k y}{2}}{\sin \frac{(N+1) y}{2}} & \text { if } y>0, \\ \frac{k}{N+1} & \text { if } y=0 .\end{cases}
$$


We have that $\phi(y)=h_{1}^{2}(y)$ and $\psi(y)=h_{N}^{2}(y)$. Differentiating $h_{k}(y)$ we find

$$
h_{k}^{\prime}(y)= \begin{cases}\frac{\frac{k}{2} \cos \frac{k y}{2} \sin \frac{(N+1) y}{2}-\frac{N+1}{2} \sin \frac{k y}{2} \cos \frac{(N+1) y}{2}}{\sin ^{2} \frac{(N+1) y}{2}} & \text { if } y>0, \\ 0 & \text { if } y=0 .\end{cases}
$$

Using the angle-sum identity $\sin (a+b)+\sin (a-b)=2 \sin a \cos b$ we rewrite this as

$$
h_{k}^{\prime}(y)= \begin{cases}\frac{\frac{N+k+1}{4} \sin \frac{(N-k+1) y}{2}-\frac{N-k+1}{4} \sin \frac{(N+k+1) y}{2}}{\sin ^{2} \frac{(N+1) y}{2}} & \text { if } y>0, \\ 0 & \text { if } y=0 .\end{cases}
$$

Define the functions $g_{k}:[0, \pi] \backslash P \rightarrow \mathbf{R}$ for each integer $k$ by

$$
g_{k}(y)=\frac{N+k+1}{4} \sin \frac{(N-k+1) y}{2}-\frac{N-k+1}{4} \sin \frac{(N+k+1) y}{2}
$$

Differentiating $g$ and simplifying using angle sum identities we find that

$$
g_{k}^{\prime}(y)=\frac{(N+1)^{2}-k^{2}}{4} \sin \frac{(N+1) y}{2} \sin \frac{k y}{2}
$$

The critical points of $g_{k}(y)$ are $y=p_{j}$ and $y=\frac{2 j \pi}{k}$. Evaluating $g_{k}(y)$ at these critical points for $k \in\{1, N\}$ we find

$$
\begin{aligned}
g_{1}\left(p_{j}\right) & =(-1)^{j+1} \frac{N+1}{2} \sin \frac{j \pi}{N+1} & g_{1}(2 j \pi) & =0, \\
g_{N}\left(p_{j}\right) & =(N+1) \cos \frac{j \pi}{N+1} & g_{N}(2 j \pi / N) & =\frac{N}{2} \cos \frac{j \pi}{N} .
\end{aligned}
$$

The function $g_{1}$ has exactly one zero in each interval $\left(p_{j-1}, p_{j}\right)$ while $h_{1}$ has no zeros in $(0, \pi)$. We conclude that $\phi$ has exactly one critical point on each interval $\left(p_{j-1}, p_{j}\right)$ and is therefore strictly convex on this interval. The function $g_{N}$ has no zeros in $(0, \pi)$ while $h_{N}$ has exactly one zero in each interval $\left(p_{j-1}, p_{j}\right)$. We conclude that $\psi$ has exactly one critical point on each interval $\left(p_{j-1}, p_{j}\right)$ and is therefore strictly convex on this interval. Each of the functions $\phi$ and $\psi$ has a critical point at $y=0$ and is therefore strictly increasing on $\left[0, p_{1}\right)$.

We prove the second part of the lemma. Let $y_{j}=\frac{2 j \pi}{2 N+1}$ for each $j \in\{1,2,3\}$. Thus

$$
\cos \frac{y_{1}}{4}>\cos \frac{y_{2}}{4} \geq \cos \frac{\pi}{5}>\frac{1}{\sqrt{2}} .
$$

Suppose $y \in\left[y_{2}, \pi\right]$. Then

$$
\phi(y) \geq \sin ^{2} \frac{y_{2}}{2}=4 \sin ^{2} \frac{y_{2}}{4} \cos ^{2} \frac{y_{2}}{4}>\frac{\sin ^{2} \frac{y_{2}}{4}}{\cos ^{2} \frac{y_{1}}{4}}=\frac{\sin ^{2} \frac{y_{1}}{2}}{\sin ^{2} \frac{(N+1) y_{1}}{2}}=\phi\left(y_{1}\right),
$$


where the second inequality follows from inequality (A.23). Now suppose $y \in\left(p_{1}, y_{2}\right)$. Evaluating $\phi$ at $y_{j}, j \in\{1,2,3\}$ we find

$$
\phi\left(y_{j}\right)=\frac{1-\cos y_{j}}{1-(-1)^{j} \cos \frac{y_{j}}{2}}=2\left(1+(-1)^{j} \cos \frac{y_{j}}{2}\right) .
$$

Thus $\phi\left(y_{2}\right)>\phi\left(y_{j}\right)$ for each $j \in\{1,3\}$. By convexity of $\phi$ on $\left(p_{1}, p_{2}\right)$ it follows that $\phi(y)>\phi\left(y_{2}\right)$ on $\left(p_{1}, y_{2}\right)$.

Acknowledgement. The author thanks Blake Temple for suggesting this problem and for his encouragement. Research supported by NSF Postdoctoral Research Fellowship, Grant Number DMS 0403188.

\section{REFERENCES}

[1] P. Baiti and H. K. Jenssen, On the front-tracking algorithm, J. Math. Anal. and Appl., 217, 395-404, 1998.

[2] A. Bressan, T. P. Liu and T. Yang, $L^{1}$ stability estimates for $n \times n$ conservation laws, Arch. Rational Mech. Anal., 149, 1-22, 1999.

[3] A. Bressan, B. Piccoli and G. Crasta, Well posedness of the Cauchy problem for $n \times n$ systems of conservation laws, Amer. Math. Soc. Memoirs, 649, 2000.

[4] R. J. Diperna, Global existence of solutions to nonlinear hyperbolic systems of conservation laws, Journal of Differential Equations, 20, 187-212, 1976.

[5] J. Glimm, Solutions in the large for nonlinear hyperbolic systems of equations, Comm. Pure Appl. Math., 18, 697-715, 1965.

[6] J. Hunter, Strongly nonlinear hyperbolic waves, Nonlinear Hyperbolic Equations - Theory, Comput. Meth. Appl., (J. Ballmann \& R.Jeltsch, ed.), Viewig, 257-268, 1989.

[7] S. N. Kruzkov, First order quasilinear equations with several space variables, Math. USSR. Sb., 10, 217-243, 1970.

[8] P. D. Lax, Hyperbolic systems of conservation laws, II, Comm. Pure Appl. Math., 10, 537-566, 1957.

[9] P. D. Lax, Shock-waves and entropy, Contributions to nonlinear functional analysis, ed. by E. Zarantonello, Academic Press, 603-634, 1971.

[10] O. A. Oleinik, Discontinuous solutions of non-linear differential equations, Uspekhi Mat. Nauk (N.S.), 12, 3-73, 1957. (Am. Math. Soc. Trans., Ser. 2, 26, 172-195).

[11] N. H. Risebro, A front tracking method for conservation laws in one dimension, J. Comp. Phys., 101, 130-139, 1992.

[12] J. Smoller, Shock Waves and Reaction-Diffusion Equations, Springer-Verlag, New York, 1982.

[13] B. Temple and R. Young, The large time stability of sound waves, Comm. Math. Phys., 179, 417-466, 1996.

[14] R. Young, Sup-norm stability for Glimm's scheme, Comm. Pure Appl. Math., 46, 903-948, 1993.

[15] R. Young, Exact solutions to degenerate conservation laws, SIAM J. Math. Anal. 30, 537-558, 1999.

[16] R. Young, Periodic solutions for conservation laws, Contemp. Math., 255, 239-256, 2000.

[17] R. Young, Sustained solutions for conservation laws, Comm. PDE, 26, 1-32, 2000. 